\title{
PPI inflation rate from $1 \%$ to $6 \%$ - Mathematical Reasoning of Economic Intervening Principle Based on Yin Yang Wu Xing Theory in Traditional Chinese Economics (II)
}

\author{
Yingshan Zhang \\ School of Statistics, Faculty of Economics and Management, \\ East China Normal University,Shanghai,P. R. China
}

\begin{abstract}
PPI (Producer Price Index) is useful in understanding economic disease. By using mathematical reasoning based on Yin Yang Wu Xing Theory in Traditional Chinese Economics (TCE), this paper demonstrates the treatment principle:"Don't have economic disease cure cure non-ill" (不治已病治未病). It means that for the PPI inflation rate of industry economic society, the normal range of theory is $[0.7362 \%, 6.4920 \%]$ nearly to $[1 \%, 6 \%]$, and the center is $3.1359 \%$ nearly to $3 \%$. The first or second transfer law of economic diseases changes according to the different PPI inflation rate whether in the normal range or not. Assume that the range of the PPI inflation rate is divided into four parts from small to large. Both second and third are for a healthy economy. The treating works are the prevention or treatment for a more serious relation economic disease which comes from the first transfer law. And both first and fourth are for an unhealthy economy.The treating works are the prevention or treatment for a more serious relation economic disease which comes from the second transfer law. Economic disease treatment should protect and maintain the balance of two incompatibility relations: the loving relationship and the killing relationship. As an application, the Chinese PPI inflation rate is used for the wood subsystem how to do works based on studying the sick subsystem of steady multilateral systems.
\end{abstract}

Keywords: Traditional Chinese Economics (TCE), Yin Yang Wu Xing Theory, steady multilateral systems, incompatibility relations, side effects, medical and drug resistance problem

\section{INTRODUCTION}

PPI (Producer Price Index) is useful in understanding industry economic disease. The main purpose in measuring all kinds of changes in price of goods in different of production. In general, the production of goods is divided into three stages: Stage one, the original: commodities have not to do any processing; Second, the intermediate stage: the commodities still needs further processing; Three, completing phase: commodity so far no longer doing any processing procedures.

PPI is changing trends and changes in prices of industrial products degree index, reflect a certain period production price movements in the field of important economic indicators, also is on economic policy and important basis of national economic accounting. At present, the investigation of PPI products are more than 4000 (including 9500 kinds of specifications), cover all 39 industry categories, types of investigated 186.

Through the growth rate of price index to calculate the rate of inflation, prices can be respectively by the consumer price index (CPI), the producer price index (PPI), the retail price index (RPI), and the gross national product (GNP) as conversion price index. In order to examine industrial development situation, general use of PPI index, its formula is as follows: 


$$
P P I=a_{1}\left(P_{1 t} / P_{10}\right)+a_{2}\left(P_{2 t} / P_{20}\right)+\ldots+a_{n}\left(P_{n t} / P_{n 0}\right),
$$

where the type of digital and $t, n$ is the number in the subscript, $P_{*}$ as the representative of Producer goods prices, $a_{n}$ is the weight.

Both the rate of PPI inflation and the PPI are two different concepts. Calculation method of the rate of PPI inflation through the calculation of the PPI changes:

$$
\begin{aligned}
& \text { The rate of PPI inflation (price rises) } \\
& =\frac{\text { current price level }- \text { base price level }}{\text { base price level }} \times 100 \% \text {, }
\end{aligned}
$$

where the price rise level from low to high, to base the level of prices for base. One of the base period is selected one price level as a reference, so that you can put the other periods of price level with a comparison between base level to measure the current level of inflation.

Note on the type, the rate of PPI inflation is not a price index, which is not a price rise, but the price index to rise. In fact, what is said above is just one of the three methods (CPI,PPI,RPI) of measuring inflation index reduced industry consumption laws, but it is the most commonly used for studying industry economy, in addition to Gross Domestic Product (GDP) and consumer price index (CPI) conversion method.

The PPI is the government measure of inflation one of the data. Popular speaking, the PPI is the price of the industry goods on the market growth percentage. As an important indicator, observe the level of inflation in China, much attention has been paid to also for such an important indicator, as a new era of youth, more objective view should be observed. First of all, let the PPI be met. The PPI is to reflect the industry products and services, related to the industry goods calculated price, industrial commodities index usually observed inflation as an important indicator.

PPI and CPI: The CPI as a consumer price index, mainly by the food and clothing live line and residents of consumer prices, the PPI as industrial factory price, is mainly composed of the prices of energy, minerals and other commodities. In between, the CPI can reflect consumer demand more, the PPI is relatively to reflect enterprise investment demand; The CPI of currency in circulation is more sensitive, while PPI relative reflect monetary velocity; Economic stimulus to the CPI conduction period is shorter, while PPI needs relatively long conduction period as well as business confidence in the economic recovery.

Su etc [1] have found that there is the bi-directional Granger dynamic causality between the PPI and the CPI. The PPI plays a key role in the CPI. So the central bank can minimize the inflation by taking certain predictive measures to keep the input prices under control.

Because of the Granger causality between the PPI and the CPI, the normal range of the PPI inflation rate can be obtainedfrom the normal range of the CPI inflation rate. It is found that the normal range of the CPI inflation rate is from $2 \%$ to $5 \%$. There are a lot of evidences (e.g., experimental identification for probability and real applications) to support this viewpoint, such as, Crone etc [2], Pauhofova etc [3],Funke etc [4], Formica etc [5], Fan etc [6], Adams [7], Hausman [8], Nahm [9], Moosa [10], Zhao [11], Daniel [12], Anonymous [13-14], and so on. 
It is believed that the normal range of the PPI inflation rate is from $1 \%$ to $6 \%$. It is because the PPI is more sensitive than the CPI, so changes in the wider range. Thus the industry economic social system identifies an important indicator for an economic social system health: the value of PPI inflation rate, which, under normal conditions, ranges from $1 \%$ to $6 \%$. Outside this range (low:Yin condition; high: Yang condition), economic disease appears. Almost always, when there is economic disease, the condition of inflation rate is a Yin condition, little is a Yang condition.

If the PPI rose is too large, it shows that the inflation has become the economic instability, the central bank will be a tight monetary policy and fiscal policy paying risk, resulting in the uncertain economic outlook. As a result, the index of high rise is often not welcome by the market. In the past 12 months, for example, the PPI roses $2.2 \%$, that means, the cost of industry making rose by an average of $2.2 \%$ more than 12 months ago. When the cost of industry making increase, your money value drops. That is to say, a 100-yuan notes, only can buy $\$ 97.70$ worth of industry goods and services. Generally when the PPI inflation rate $>1 \%$, it is called plus, is INFLATION. And when the PPI inflation rate $>6 \%$, it is treated as a SERIOUS plus, is a SERIOUS INFLATION.

In this paper, the rate of inflation can be considered as the price level rises rather than the currency quantity rises from the basic concept of PPI. It is because the PPI is the direct reflection of industry making standards, although the price level increase is difficult to be controlled directly.

The PPI is a general parameter linking together the complexity of relations between subsystem pairs of economic social system, economic social system itself, the capabilities for intervention reaction and self-protection of the economic social system as an economy and mind as a whole, related to the environment, food, health and personal history, air, water, earth, climate, season, etc. PPI is as useful in understanding economic disease as the average is in statistics, or as the expected value is in probability calculation.

The economic social system as an economy begins to activate the necessary mechanisms to restore this parameter to its appropriate range. If the economic social system as an economy is unable to restore optimal PPI levels, the economic disease may become chronic and lead to dire consequences.

Zhang etc [15-23] have started a great interest and admired works for Traditional Chinese Economics (TCE), where, through mathematical reasoning, they demonstrate the presence of incompatibility relations, which are predominant in daily life, yet absent in traditional Aristotelian Western logic.

Many people as Western persons are beyond all doubt the Yin Yang Wu Xing theory is superior to the traditional true-false logic, which does not contemplate incompatibility relations, which Zhang [19] has expertly explained from a mathematical standpoint.

The work Zhang $[15,16]$ has started, allows many people like Western person to think of a true re-foundation of mathematical language, to make it a better suited tool for the needs of mankind economic social system and the environment. Although so doing, Zhang [18] also brings to light the difficulty of establishing the values of both the intervention reaction coefficients $\rho_{1}, \rho_{2}$ and the self-protection coefficient $\rho_{3}$ as parameters with due accuracy. 
In this paper, the introduction of a parameter such as a PPI will be suggested, in order to facilitate the understanding and the calculation of the values of both the intervention reaction coefficients $\rho_{1}, \rho_{2}$ and the self-protection coefficient $\rho_{3}$. This paper ventures to suggest this with all due to respect, because it be believed that the path Zhang $[15,16]$ has started, in such an understandable way from the mathematical point of view, will be very useful for all mankind searching for tools to understand the mechanisms of economic social system.

The article proceeds as follows. Section 2 contains a parameter model and basic theorems, in order to explain both the intervention reaction coefficients $\rho_{1}, \rho_{2}$ and the self-protection coefficient $\rho_{3}$ through the introduction of a parameter model to study the normal range of a PPI inflation rate, while the first or second transfer law of economic diseases is demonstrated in Section 3, proved through the concept of both relation diseases and a relationship analysis of steady multilateral systems. Furthermore, if the range of PPI inflation rate is divided into four parts, for the economy in every part, the prevention or treatment method of economic diseases as treatment principle of TCE is given in Section 4. As an application, the Chinese PPI for studying the $\operatorname{wood}(X)$ subsystem of steady multilateral systems in Section 5 and conclusions are drawn in Section 6.

\section{PARAMETER MODEL AND BASIC THEOREMS}

The concepts and notations in Zhang [21] are start and still used.

Let $\varphi=(\sqrt{5}-1) / 2=0.61803399$ be the gold number. Denoted $\rho_{0}=0.5897545123$, namely healthy number. It is because the healthy number $\rho_{0}$ can make the healthy balance conditions $\rho_{1}=\rho_{3}$, $\rho_{2}=\rho_{1} \rho_{3}$ and $1-\rho_{2} \rho_{3}=\rho_{1}+\rho_{2} \rho_{3}$ hold if $\rho_{1}=\rho_{0}, \rho_{2}=\rho_{0}^{2}$ and $\rho_{3}=\rho_{0}$.

Assuming $\rho_{0}^{\prime}=0.68232780$, namely unhealthy number. It is because under a poor self-protection ability, the unhealthy number $\rho_{0}^{\prime}$ can make the poor healthy balance conditions hold:

$$
\begin{gathered}
\rho_{1}-\rho_{3}=\rho_{3}=\rho_{0}^{\prime} / 2=0.34116390, \\
\rho_{2}-\rho_{1} \rho_{3}=\rho_{1} \rho_{3}=\left(\rho_{0}^{\prime}\right)^{2} / 2=0.23278561 \\
1-\rho_{2} \rho_{3}=\rho_{1}+\rho_{2} \rho_{3} \\
\text { if } \rho_{1}=\rho_{0}^{\prime}, \rho_{2}=\left(\rho_{0}^{\prime}\right)^{2}=0.46557123 \text { and } \rho_{3}=\frac{1}{2} \rho_{0}^{\prime} \text {. Thus } \rho_{0}<\varphi<\rho_{0}^{\prime} .
\end{gathered}
$$

A parameter model of a PPI inflation rate in a mathematical sense based on Yin Yang Wu Xing Theory of TCE is reintroduced by using the functions $\lambda(x)$ and $\rho(x)$ of the PPI inflation rate $x$ described as follows.

Let $x \in(-0.1,0.65)$ be a PPI inflation rate, where the values -0.1 and 0.65 are the minimum and maximum acceptable the PPI inflation rate. Denoted the value 0.031359 is the target as the expectation of the PPI inflation rate. Define a function $\lambda(x)$ of the PPI inflation rate $x$ in below:

$$
\begin{aligned}
& \lambda(x)=\frac{|x-0.031359|}{(0.65-x)(x+0.1)}, \forall x \in(-0.1,0.65) \\
& = \begin{cases}\frac{x-0.031359}{(0.65-x)(x+0.1)}, & 0.6>x \geq 0.031359 ; \\
\frac{0.031359-x}{(0.65-x)(x+0.1)}, & -0.1<x<0.031359 .\end{cases}
\end{aligned}
$$

A parameter model is considered as 


$$
\rho(x)=\frac{1 / 2}{\lambda(x)+1 / 2}, \forall x \in(-0.1,0.65)
$$

\section{Theorem 2.1 Under model (4), the following statements hold.}

(1) The one that

$$
0<\rho(x)=\frac{1 / 2}{\lambda(x)+1 / 2} \leq 1 \text { is equivalent to the other that } 0 \leq \lambda(x)=\frac{1-\rho(x)}{2 \rho(x)}<+\infty,
$$

where $\lambda(x)$ is a monotone decreasing function of $x$ if $x \in(-0.1,0.031359]$ or a monotone increasing function of $x$ if $x \in[0.031359,0.65)$; and $\rho(x)$ is a monotone decreasing function of $\lambda(x)$ if $\lambda(x) \in[0,+\infty)$; and $\lambda(x)$ is a monotone decreasing function of $\rho(x)$ if $\rho(x) \in(0,1]$.

(2) If $1 \geq \rho(x) \geq \rho_{0}$, then $\lambda(x)=\frac{1-\rho(x)}{2 \rho(x)} \leq \frac{1-\rho_{0}}{2 \rho_{0}}=\rho_{0}^{2} \leq \rho(x)^{2} \leq 1$;

$\frac{\lambda(x)}{\rho(x)}=\frac{1-\rho(x)}{2 \rho(x)^{2}} \leq \frac{1-\rho_{0}}{2 \rho_{0}^{2}}=\rho_{0} \leq \rho(x) \leq 1 ;$ and $\frac{\lambda(x)}{\rho(x)^{2}}=\frac{1-\rho(x)}{2 \rho(x)^{3}} \leq \frac{1-\rho_{0}}{2 \rho_{0}^{3}}=1$.

(3) If $0<\rho(x)<\rho_{0}$, then

$$
\lambda(x)=\frac{1-\rho(x)}{2 \rho(x)}>\frac{1-\rho_{0}}{2 \rho_{0}}=\rho_{0}^{2}>\rho(x)^{2}>0 ;
$$

$$
\frac{\lambda(x)}{\rho(x)}=\frac{1-\rho(x)}{2 \rho(x)^{2}}>\frac{1-\rho_{0}}{2 \rho_{0}^{2}}=\rho_{0}>\rho(x)>0 ; \text { and } \frac{\lambda(x)}{\rho(x)^{2}}=\frac{1-\rho(x)}{2 \rho(x)^{3}}>\frac{1-\rho_{0}}{2 \rho_{0}^{3}}=1 .
$$

(4) Taking $0<\rho_{1}=\rho(x)<\rho_{0}, \rho_{2}=\rho(x)^{2}$ and $\rho_{3}=c \rho(x)$ where $0 \leq c \leq 1$, there are $\rho_{1}-\rho_{3}=\rho(x)(1-c) \geq 0, \rho_{2}-\rho_{1} \rho_{3}=\rho(x)^{2}(1-c) \geq 0$, and $\left(\rho_{1}+\rho_{2} \rho_{3}\right)=\rho(x)+c \rho(x)^{3}<1-\rho_{2} \rho_{3}=1-c \rho(x)^{3}$, where $\left|\left(\rho_{1}+\rho_{2} \rho_{3}\right)-\left(1-\rho_{2} \rho_{3}\right)\right|>2(1-c) \rho_{0}^{3}=(1-c) 0.41024$. (5) Taking $1 \geq \rho_{1}=\rho(x) \geq \rho_{0}, \rho_{2}=\rho(x)^{2} \quad$ and $\rho_{3}=c \rho(x)$ where $0 \leq c \leq 1$, there are

firstly, $\rho_{1}-\rho_{3}=\rho(x)(1-c) \geq 0, \rho_{2}-\rho_{1} \rho_{3}=\rho(x)^{2}(1-c) \geq 0$ and $\left(\rho_{1}+\rho_{2} \rho_{3}\right)=\rho(x)+c \rho(x)^{3} \geq 1-\rho_{2} \rho_{3}=1-c \rho(x)^{3}$ if $1 \geq c \geq \frac{1-\rho(x)}{2 \rho(x)^{3}}=\frac{\lambda(x)}{\rho(x)^{2}} \geq 0 ;$

secondly, $\rho_{1}-\rho_{3}=\rho(x)(1-c)>\rho(x) / 2, \rho_{2}-\rho_{1} \rho_{3}=\rho(x)^{2}(1-c)>\rho(x)^{2} / 2$ and $\left(\rho_{1}+\rho_{2} \rho_{3}\right)=\rho(x)+c \rho(x)^{3}<1-\rho_{2} \rho_{3}=1-c \rho(x)^{3} \quad$ where this inequality range to meet $\left|\left(\rho_{1}+\rho_{2} \rho_{3}\right)-\left(1-\rho_{2} \rho_{3}\right)\right| \leq\left(\rho_{0}^{\prime}\right)^{3}=0.31767$ if $0 \leq c<\frac{1-\rho(x)}{2 \rho(x)^{3}}=\frac{\lambda(x)}{\rho(x)^{2}} \leq \frac{1}{2}$ in which $1>\rho(x) \geq \rho_{0}^{\prime}$;

thirdly, $\rho_{1}-\rho_{3}=\rho(x)(1-c) \geq \rho(x) / 2, \rho_{2}-\rho_{1} \rho_{3}=\rho(x)^{2}(1-c) \geq \rho(x)^{2} / 2$ and $\left(\rho_{1}+\rho_{2} \rho_{3}\right)=\rho(x)+c \rho(x)^{3}<1-\rho_{2} \rho_{3}=1-c \rho(x)^{3}$ where this inequality range to meet $\left|\left(\rho_{1}+\rho_{2} \rho_{3}\right)-\left(1-\rho_{2} \rho_{3}\right)\right| \leq 2 \rho_{0}^{3}=0.41024$ if $0 \leq c \leq \frac{1}{2}<\frac{1-\rho(x)}{2 \rho(x)^{3}}=\frac{\lambda(x)}{\rho(x)^{2}} \leq 1$ in which $\rho_{0} \leq \rho(x)<\rho_{0}^{\prime}$;

finally, $\rho_{1}-\rho_{3}=\rho(x)(1-c)<\rho(x) / 2, \rho_{2}-\rho_{1} \rho_{3}=\rho(x)^{2}(1-c)<\rho(x)^{2} / 2$ and $\left(\rho_{1}+\rho_{2} \rho_{3}\right)=\rho(x)+c \rho(x)^{3}<1-\rho_{2} \rho_{3}=1-c \rho(x)^{3}$ where this inequality range to meet $\left|\left(\rho_{1}+\rho_{2} \rho_{3}\right)-\left(1-\rho_{2} \rho_{3}\right)\right|<\left(\rho_{0}^{\prime}\right)^{3}=0.31767$ if $\frac{1}{2}<c<\frac{1-\rho(x)}{2 \rho(x)^{3}}=\frac{\lambda(x)}{\rho(x)^{2}} \leq 1$ in which $\rho_{0} \leq \rho(x)<\rho_{0}^{\prime}$.

In particular, when $c$ is nearly to $1 / 2$, there are $\rho_{1}-\rho_{3}=\rho(x)(1-c) \rightarrow \rho(x) / 2, \rho_{2}-\rho_{1} \rho_{3}=\rho(x)^{2}(1-c) \rightarrow \rho(x)^{2} / 2$ and the following statements hold. 
(a). The absolute value $\left|\left(\rho_{1}+\rho_{2} \rho_{3}\right)-\left(1-\rho_{2} \rho_{3}\right)\right|$ is nearly to 0 if $0<c<\frac{1-\rho(x)}{2 \rho(x)^{3}}=\frac{\lambda(x)}{\rho(x)^{2}} \leq \frac{1}{2}$ in which $1>\rho(x) \geq \rho_{0}^{\prime}$.

(b). The value $\left[\left(\rho_{1}+\rho_{2} \rho_{3}\right)-\left(1-\rho_{2} \rho_{3}\right)\right]$ is included in the interval $\left[-\rho_{0}^{3}=-0.20512,0\right)$ respectively if $0<c \leq \frac{1}{2}<\frac{1-\rho(x)}{2 \rho(x)^{3}}=\frac{\lambda(x)}{\rho(x)^{2}} \leq 1$ in which $\rho_{0} \leq \rho(x)<\rho_{0}^{\prime}$.

(c).The value $\left[\left(\rho_{1}+\rho_{2} \rho_{3}\right)-\left(1-\rho_{2} \rho_{3}\right)\right]$ is included in the interval $\left[-\rho_{0}^{3}=-0.20512,0\right)$ respectively if $\frac{1}{2}<c<\frac{1-\rho(x)}{2 \rho(x)^{3}}=\frac{\lambda(x)}{\rho(x)^{2}} \leq 1$ in which $\rho_{0} \leq \rho(x)<\rho_{0}^{\prime}$. \# Corollary 2.1 Under model (4), the following statements hold.

(1) For any $0<d<1$, there is an unique solution $u \in(-0.1,0.031359)$ and there is also an unique solution $v \in(0.031359,0.65)$, such that

$\lambda(0.031359)=0 \leq \lambda(x)=\frac{1-\rho(x)}{2 \rho(x)} \leq \lambda(u)=\lambda(v)=(1-d) /(2 d)$,

$\rho(u)=\rho(v)=d \leq \rho(x)=\frac{1 / 2}{\lambda(x)+1 / 2} \leq 1=\rho(0.031359)$.

(2) The condition $x \in[0.01,0.06]$ is equivalent to each of the following conditions:

$\lambda(0.031359)=0 \leq \lambda(x)=\frac{1-\rho(x)}{2 \rho(x)} \leq \lambda(0.01)=\lambda(0.06)=0.30340$,

$\rho(0.01)=\rho(0.06)=0.62236 \leq \rho(x)=\frac{1 / 2}{\lambda(x)+1 / 2} \leq 1=\rho(0.031359)$.

(3) The condition $x \in[0.009660,0.060612]$ is equivalent to each of the following conditions:

$\lambda(0.031359)=0 \leq \lambda(x)=\frac{1-\rho(x)}{2 \rho(x)} \leq \lambda(0.009660)=\lambda(0.060612)=\frac{1-\varphi}{2 \varphi}=0.30902$,

$\rho(0.009660)=\rho(0.060612)=\varphi \leq \rho(x)=\frac{1 / 2}{\lambda(x)+1 / 2} \leq 1=\rho(0.031359)$.

(4) The condition $x \in[0.007362,0.064920]$ is equivalent to each of the following conditions:

$\lambda(0.031359)=0 \leq \lambda(x)=\frac{1-\rho(x)}{2 \rho(x)} \leq \lambda(0.007362)=\lambda(0.064920)=\rho_{0}^{2}=0.34781$,

$\rho(0.007362)=\rho(0.064920)=\rho_{0} \leq \rho(x)=\frac{1 / 2}{\lambda(x)+1 / 2} \leq 1=\rho(0.031359)$.

(5) The condition $x \in[0.014429,0.052578]$ is equivalent to each of the following conditions: $\lambda(0.031359)=0 \leq \lambda(x) \leq \lambda(0.014429)=\lambda(0.052578)=\left(\rho_{0}^{\prime}\right)^{2} / 2=0.23279$,

$\rho(0.014429)=\rho(0.052578)=\rho_{0}^{\prime} \leq \rho(x)=\frac{1 / 2}{\lambda(x)+1 / 2} \leq 1=\rho(0.031359)$. \#

Remark 1. In west, through experiment or through practice observation, by using the Granger causality between the PPI and the CPI, many researchers [1-14] can obtain the normal range of the PPI inflation rate as $x \in[0.01,0.06]$ from the normal range of the CPI as $x \in[0.02,0.05]$. But in TCE, from Yin Yang Wu Xing Theory, Zhang (2013) [21] has already determined: $\rho_{0} \leq \rho_{1} \leq 1$ for the normal range of a healthy economy. Taking $\rho_{1}=\rho(x), \rho_{2}=\rho(x)^{2}$ and $\rho_{3}=c \rho(x)$ where $0 \leq c \leq 1$ for an economic society which has the capabilities of both intervention reaction and selfprotection. From Corollary 2.1, the condition $\rho_{0} \leq \rho_{1} \leq 1$ is equivalent to that $x \in[0.007362,0.064920]$. In other words, in Theory of TCE, the normal range of the PPI inflation rate is considered as 
$x \in[0.007362,0.064920]$, nearly to $x \in[0.01,0.06]$. Of course, little difference of the two intervals which makes the diagnosis of disease as a result, there may be no much difference as a suspect. In fact, TCE uses the rule $\rho_{0} \leq \rho_{1} \leq 1$ from Yin Yang Wu Xing Theory instead of the normal range of a PPI inflation rate. The equivalence of Corollary 2.1 shows that TCE is The scientific which is from TCM (Traditional Chinese Medicine).

Zhang etc [21] has already determined: an economy is said a healthy mathematical complex system when the intervention reaction coefficient $\rho_{1}$ satisfies $1 \geq \rho_{1} \geq \rho_{0}$. In logic and practice, it's reasonable that $\rho_{1}+\rho_{2}$ is near to 1 if the input and output in a complex system is balanced, since a mathematical output subsystem is absolutely necessary other subsystems of all consumption. In case: $\rho_{1}+\rho_{2}=1$, all the energy for intervening mathematical complex subsystem can transmit to other mathematical complex subsystems which have neighboring relations or alternate relations with the intervening mathematical complex subsystem. The condition $\rho_{1} \geq \rho_{0}$ can be satisfied when $\rho_{2}=\rho_{1} \rho_{3}$ and $\rho_{3}=\rho_{1}$ for a mathematical complex system since $\rho_{1}+\rho_{2}=1$ implies $\rho_{1}=\varphi \approx 0.61803 \geq \rho_{0}$. In this case, $\rho_{2}=\varphi^{2} \approx 0.38197$. If this assumptions is set up, then the intervening principle: "Real disease with a healthy economy is to rush down its son and virtual disease with a healthy economy is to fill its mother" based on "Yin Yang Wu Xing" theory in image mathematics [20] , is quite reasonable. But, in general, the ability of self-protection often is insufficient for an usual mathematical complex system, i.e., $\rho_{3}$ is small. A common standard is $\rho_{3}=\frac{1-\rho_{1}}{2 \rho_{2}} \approx \frac{1}{2}$ which comes from the balance condition $\left(1-\rho_{2} \rho_{3}\right)=\left(\rho_{1}+\rho_{2} \rho_{3}\right)$ of the loving relationship if $\rho_{1}+\rho_{2} \approx 1$. In other words, there is a principle which all losses are bear in mathematical complex system. Thus the general condition is often $\rho_{1} \approx 0.61803 \geq \rho_{3} \approx 0.5 \geq \rho_{2} \approx 0.38197$. Interestingly, they are all near to the golden numbers. It is the idea to consider the unhealthy number $\rho_{0}^{\prime}=0.68232780$ since the poor condition of selfprotection ability $\rho_{3}=\rho_{1} / 2=\rho_{0}^{\prime} / 2=0.34116390$ can make the following unhealthy balance conditions holding

$$
\begin{aligned}
& \rho_{1}-\rho_{3}=\rho_{3}=\rho_{0}^{\prime} / 2=0.34116390, \\
& \rho_{2}-\rho_{1} \rho_{3}=\rho_{1} \rho_{3}=\left(\rho_{0}^{\prime}\right)^{2} / 2=0.23278561 \\
& 1-\rho_{2} \rho_{3}=\rho_{1}+\rho_{2} \rho_{3} \\
& \text { if } \rho_{1}=\rho_{0}^{\prime} \text { and } \rho_{2}=\left(\rho_{0}^{\prime}\right)^{2}=0.46557123 .
\end{aligned}
$$

By Theorem 2.1 and Corollary 2.1, the interval $x \in[0.01,0.06]$ implies the following condition $1 \geq \rho_{1}=\rho(x) \geq 0.62236=\rho(0.01)=\rho(0.06)$;

and the interval $x \in[0.009660,0.060612]$ implies the following condition $1 \geq \rho_{1}=\rho(x) \geq \varphi=\rho(0.009660)=\rho(0.060612)$;

and the interval $x \in[0.007362,0.064920]$ implies the following condition $1 \geq \rho_{1}=\rho(x) \geq \rho_{0}=\rho(0.007362)=\rho(0.064920)$,

where $\lambda(0.007362)=\lambda(0.007362)=\frac{1-\rho_{0}}{2 \rho_{0}}=\rho_{0}^{2}$ since $\left(1-\rho_{0}^{3}\right)=\left(\rho_{0}+\rho_{0}^{3}\right)$; and the interval $x \in[0.014429,0.052578]$ implies the following condition $1 \geq \rho_{1}=\rho(x) \geq \rho_{0}^{\prime}=\rho(0.014429)=\rho(0.052578)$, 
where

$\lambda(0.014429)=\lambda(0.052578)=\frac{1-\rho_{0}^{\prime}}{2 \rho_{0}^{\prime}}=\frac{\left(\rho_{0}^{\prime}\right)^{2}}{2}$ since $\left(\rho_{0}^{\prime}\right)^{3}=\left(1-\rho_{0}^{\prime}\right)$.

The last one is the healthy interval in an economic society's self-protection ability poor conditions. The interval range than the normal economic society health requirements is too strict, only the first three interval ranges can be considered as a normal economic society health. If keep two decimal places, then first three intervals are the same as $x \in[0.01,0.06]$. This shows that range $x \in[0.01,0.06]$ is stable. The interval as the normal range of a PPI inflation rate may be also appropriate. To conservative estimates, one of the first three interval ranges with the largest length can be used, i.e., $x \in[0.007362,0.064920]$, as the theoretical analysis of the normal range in this paper. In fact, the range $x \in[0.007362,0.064920]$ is better than the range $x \in[0.01,0.06]$ because $\rho_{0}=\rho(0.007362)=\rho(0.064920)$ and $\lambda(0.007362)=\lambda(0.064920)=\frac{1-\rho_{0}}{2 \rho_{0}}=\rho_{0}^{2}$, which satisfy the healthy balance conditions $\rho_{1}=\rho_{3}, \rho_{2}=\rho_{1} \rho_{3}$, and $\left(1-\rho_{2} \rho_{3}\right) \leq\left(\rho_{1}+\rho_{2} \rho_{3}\right)$ at the same time if $\rho_{1}=\rho_{0}, \rho_{2}=\rho_{0}^{2}$ and $\rho_{3}=c \rho_{0}$ where $c \rightarrow 1$. In other words, the parameter $\rho_{1}=\rho(x) \geq \rho_{0}$ or the range $x \in[0.007362,0.064920]$ is the healthy condition of both the killing relationship and the loving relation at the same time. But neither are the others. The PPI inflation rate must be precise calculation to keep at least 6 decimal places can ensure correct because of its sensitivity to the diagnosis of disease.

Remark 2. Western Economics is different from TCE because the TCE has a concept of Chi or Qi as a form of energy. From the energy concept, that one organ or subsystem of the economic society is not running properly (or disease, abnormal), is that the energy deviation from the average of the organ is too large, the high (real disease) or the low (virtual disease). But there do not exist these concepts of both real diseases and virtual diseases in Western science. For the normal range of a PPI inflation rate of some economic society as $x \in[0.007362,0.064920]$, in TCE, if $x>0.064920$, the economy is considered as a real disease since the PPI inflation rate is too high; if $x<0.007362$, the economy is considered as a virtual disease since the PPI inflation rate is too low. Thus TCE identifies an important indicator for an economic society's health: the value of the PPI inflation rate, which, under normal conditions, ranges from 0.007362 to 0.064920 . Outside this range (too low: Yin condition; too high: Yang condition), disease appears. Almost always absolutely, when there is a virtual disease, the condition of the PPI inflation rate is a Yin condition; when there is a real disease, the condition of the PPI inflation rate is a Yang condition.

Remark 3. Obviously, when applying the hypothesis of Theorem $\mathbf{2 . 1}$ and Corollary $\mathbf{2 . 1}$ to other fields rather than economic society's health, it is necessary to identify a global parameter in each field that is able to yield a general Yin or Yang condition in relation to the average behavior of the studied phenomenon, and that maintains the equations at a sufficiently simple level of writing and application. In fact, let $x \in(\min , \max )$ where the values $\min$ and $\max$ are the minimum and maximum acceptable the index $x$. Denoted the value $t_{0}$ is the target as the expectation of the index $x$ such that $\rho\left(t_{0}\right)=1$. In Eqs.(3) and (4), replace $-0.1,0.65,0.031359$ by $\min , \max , t_{0}$, respectively. The equivalent condition of a healthy economy $\rho_{0} \leq \rho_{1}=\rho(x) \leq 1$ can be obtained as $\quad x \in[u, v], \min <u<t_{0}<v<\max , \quad$ where $\rho(u)=\rho(v)=\rho_{0} \leq \rho_{1}=\rho(x)=(1 / 2) /[\lambda(x)+(1 / 2)] \leq \rho\left(t_{0}\right)=1$ and $\lambda\left(t_{0}\right)=0 \leq \lambda(x)=(1-\rho(x)) /(2 \rho(x)) \leq \rho_{0}^{2}=\lambda(u)=\lambda(v)=\rho(u)^{2}=\rho(v)^{2} \leq \rho_{2}=\rho(x)^{2}$ $\leq \rho_{1}=\rho(x) \leq 1$.\# 


\subsection{Energy Changes of a Multilateral System}

\section{RELATIONS OF STEADY MULTILATERAL SYSTEMS}

In order to apply the reasoning to other fields rather than society economy's health, Zhang etc [21] have started a steady multilateral system imitating society economy. A most basic steady multilateral system is as follows .

Theorem 3.1 [20] For each element $x$ in a steady multilateral system $v$ with two incompatibility relations, there exist five equivalence classes below:

$$
\begin{gathered}
X=\{y \in V \mid y \sim x\}, X_{S}=\{y \in V \mid x \rightarrow y\}, X_{K}=\{y \in V \mid x \Rightarrow y\}, \\
K_{X}=\{y \in V \mid y \Rightarrow x\}, S_{X}=\{y \in V \mid y \rightarrow x\},
\end{gathered}
$$

which the five equivalence classes have relations in Figure 1. \#

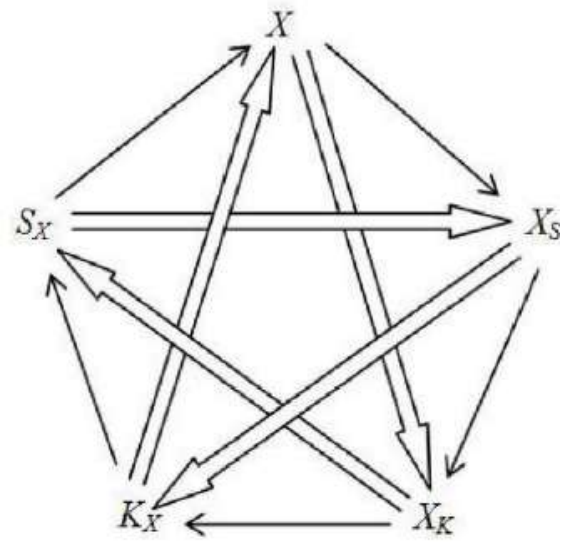

Figure 1. Finding Yin Yang Wu Xing Model

It can be proved by Theorem 3.2 below that the steady multilateral system in Theorem 3.1 is the reasoning model of Yin Yang Wu Xing in TCE if there is an energy function $\varphi(*)$ satisfying

$$
\varphi\left(X_{K}\right) \geq \varphi\left(X_{S}\right) \geq \varphi(X) \geq \varphi\left(K_{X}\right) \geq \varphi\left(S_{X}\right) .
$$

It is called Yin Yang Wu Xing model, denoted by $V^{5}$.

The Yin Yang Wu Xing model can be written as follows: Define

$$
V_{0}=X, V_{1}=X_{S}, V_{2}=X_{K}, V_{3}=K_{X}, V_{4}=S_{X},
$$

corresponding to wood, fire, earth, metal, water, respectively, and assume $V=V_{0}+V_{1}+V_{2}+V_{3}+V_{4}$ where $V_{i} \cap V_{j}=\varnothing, \forall i \neq j$.

And take $\mathfrak{R}=\left\{R_{0}, R_{1}, \cdots, R_{4}\right\}$ satisfying $R_{r}=\sum_{i=0}^{4} V_{i} \times V_{\bmod (i+r, 5)}, \forall r \in\{0,1, \cdots, 4\}, R_{i}^{*} * R_{j} \subseteq R_{\bmod (i+j, 5)}$,

where $V_{i} \times V_{j}=\left\{(x, y): x \in V_{i}, y \in V_{j}\right\}$ is the Descartes product in set theory and $R_{i} * R_{j}=\left\{(x, y): \exists u \in V\right.$ such that $\left.(x, u) \in R_{i},(u, y) \in R_{j}\right\}$ is the multiplication relation operation. The relation multiplication of $*$ is isomorphic to the addition of module 5 . Then $V^{5}$ is a steady multilateral system with one equivalent relation $R_{0}$ and two incompatibility relations $R_{1}=R_{4}^{-1}$ and $R_{2}=R_{3}^{-1}$ where $R_{i}^{-1}=\left\{(x, y):(y, x) \in R_{i}\right\}$ is the inverse operation. The Yin and Yang 
means the two incompatibility relations and the $\mathrm{Wu}$ Xing means the collection of five disjoint classification of $V=V_{0}+V_{1}+V_{2}+V_{3}+V_{4}$.

Figure 1 in Theorem 3.1 is the Figure of Yin Yang Wu Xing theory in Ancient China. The steady multilateral system $V$ with two incompatibility relations is equivalent to the logic architecture of reasoning model of Yin Yang Wu Xing theory in Ancient China. What describes the general method of complex systems can be used in society complex systems.

By non-authigenic logic of TCE, i.e., a logic which is similar to a group has nothing to do with the research object [20], in order to ensure the reproducibility such that the analysis conclusion can be applicable to any complex system, a logical analysis model which has nothing to do with the object of study should be chosen. The Tao model of Yin and Yang is a generalized one which means that two is basic. But the Tao model of Yin Yang is simple in which there is not incompatibility relation. The analysis conclusion of Tao model of Yin Yang cannot be applied to an incompatibility relation model. Thus the Yin Yang Wu Xing model with two incompatibility relations of Theorem $\mathbf{3 . 1}$ will be selected as the logic analysis model in this paper.

Western Economy is different from TCE because the TCE has a concept of Chi or Qi (气) as a form of energy of steady multilateral systems. It is believed that this energy exists in all things of steady multilateral systems (living and non-living) including air, water, food and sunlight. Chi is said to be the unseen vital force that nourishes steady multilateral systems' economy and sustains steady multilateral systems' life. It is also believed that an individual is born with an original amount of Chi at the beginning of steady multilateral systems' life and as a steady multilateral system grows and lives, the steady multilateral system acquires or attains Chi or energy from "eating" and "drinking", from "breathing" the surrounding "air" and also from living in its environment. The steady multilateral system having an energy is called the anatomy system or the first physiological system. And the first physiological system also affords Chi or energy for the steady multilateral system's meridian system (Jing-Luo (经络) or Zang Xiang (藏象)) which forms a parasitic system of the steady multilateral system, called the second physiological system of the steady multilateral system. The second physiological system of the steady multilateral system controls the first physiological system of the steady multilateral system. A steady multilateral system would become ill or dies if the Chi or energy in the steady multilateral system is imbalanced or exhausted, which means that $\rho_{1}=\rho(x) \rightarrow 0, \rho_{2}=\rho(x)^{2} \rightarrow 0$ and $\rho_{3}=c \rho(x) \rightarrow 0$.

For example, in TCE, a society economy as the first physiological system of the steady multilateral system following the Yin Yang Wu Xing theory was classified into five equivalence classes as follows:

$\operatorname{wood}(x)=\{$ industry, PPI (the Producer Price Index) or RPI (Retail Price Index), liver, bravery, soul, ribs, sour, east, spring, birth\};

xiang-fire $\left(X_{S}^{x}\right)=$ agriculture, AAF (the total output value of Agriculture forestry Animal husbandry and Fishery) , pericardium, the triple energizer, nerve, the blood, bitter taste, the south, summer, growth\};

$\operatorname{earth}\left(X_{K}\right)=\{$ commerce, CPI (the Consumer Price Index), spleen, stomach, willing, meat, sweetness, center, long summer, combined\}; 
$\operatorname{metal}\left(K_{x}\right)=\{$ science-education, GBR (the General Budget Revenue), lung, large intestine, boldness, fur, spicy, west, autumn, accept\};

water $\left(s_{x}\right)=\{$ army-economic,GDP (the Gross Domestic Product), kidney, bladder, ambition, bone, salty, the north, winter, hiding\};

jun-fire $\left(X_{S}^{j}\right)=\{$ President or Governor, Finance (right of making money), heart, small intestine, bitter taste, whole economy, throughout the year, overall growth\}.

fire $\left(X_{S}\right)=$ xiang-fire $\left(X_{S}^{x}\right) \cup$ jun-fire $\left(X_{S}^{j}\right)$.

There is only one of both loving and killing relations between every two classes. General close is loving, alternate is killing.

In every category of internal, think that they are with an equivalent relationship, between each two of their elements there is a force of similar material accumulation of each other. It is because their pursuit of the goal is the same, i.e., follows the same "Axiom system". It can increase the energy of the class at low cost near to zero if they accumulate together. Any nature material activity follows the principle of maximizing so energy or minimizing the cost. In general, the size of the force of similar material accumulation of each other is smaller than the size of the loving force or the killing force in a stable complex system. The stability of any complex system first needs to maintain the equilibrium of the killing force and the loving force. The key is the killing force. For a stable complex system, if the killing force is large, i.e., $\rho_{3}=c \rho(x)$ becomes larger by Theorem 3.3 below, which needs positive exercise, then the loving force is also large such that the force of similar material accumulation of each other is also large. They can make the complex system more stable. If the killing force is small, i.e., $\rho_{3}=c \rho(x)$ becomes smaller by Theorem 3.3 below, which means little exercise, then the loving force is also small such that the force of similar material accumulation of each other is also small. They can make the complex system becoming unstable.

The second physiological system of the steady multilateral system controls the first physiological system of the steady multilateral system, abiding by the following rules.

Attaining Rule: The second physiological system of the steady multilateral system will work by using Attaining Rule, if the first physiological system of the steady multilateral system runs normally. The work is in order to attain the Chi or energy from the first physiological system of the steady multilateral system by mainly utilizing the balance of the loving relationship of the first physiological system.

In mathematics, suppose that the economy of $x$ is healthy. If $x$ is intervened, then the second physiological system will attain the Chi or energy from $x$ directly.

Suppose that the economy of $x$ is unhealthy. If $x$ is intervened, then the second physiological system will attain the Chi or energy from $x$ indirectly. If virtual $x$ is intervened, it will attain the Chi or energy (Yang energy) from the son $x_{S}$ of $x$. If real $x$ is intervened, it will attain the Chi or energy (Yin energy) from the mother $s_{X}$ of $x$.\#

Affording Rule: The second physiological system of the steady multilateral system will work by using Affording Rule, if the first physiological system of the steady multilateral system runs hardly. The work is in order to afford the Chi or energy for the first physiological system of the steady multilateral system, by mainly protecting or maintaining the balance of the killing 
relationship of the steady multilateral system, to drive the first physiological systems will begin to run normally.

In mathematics, suppose that the economy of $X$ is healthy. The second physiological system doesn't afford any Chi or energy for the first physiological system.

Suppose that the economy of $X$ is unhealthy and the capability of self-protection is lack, i.e., $\rho_{3}=c \rho(x) \rightarrow 0$ and $0<\rho_{1}=\rho(x)<\rho_{0}$. The second physiological system will afford the Chi or energy for $X$ directly, at the same time, affording the Chi or energy for other subsystem, in order to protect or maintain the balance of the killing relationship, abiding by the intervening principle of "Strong inhibition of the same time, support the weak", such that the capability of selfprotection is restored, i.e, $\rho_{3}=c \rho(x)>0$ and $1 \geq \rho_{1}=\rho(x) \geq \rho_{0}$, to drive the first physiological system beginning to work.\#

The Chi or energy is also called the food hereafter for simply. In order to get the food, by Attaining Rule, the second physiological system must make the first physiological system intervened, namely exercise. It is because only by intervention on the first physiological system, the second physiological system is able to get food.

Energy concept is an important concept in Physics. Zhang [17] introduces this concept to the steady multilateral systems or image mathematics [20] and uses these concepts to deal with the steady multilateral system diseases (mathematical index too high or too low). In mathematics, a steady multilateral system is said to have Energy (or Dynamic) if there is a non-negative function $\varphi(*)$ which makes every subsystem meaningful of the steady multilateral system. Similarly to Zhang [18], unless stated otherwise, any equivalence relation is the liking relation, any neighboring relation is the loving relation, and any alternate relation is the killing relationship.

Suppose that $v$ is a steady multilateral system having an energy, then $v$ in the steady multilateral system during a normal operation, its energy function for any subsystem of the steady multilateral system has an average (or expected value in Statistics), this state is called as normal when the energy function is nearly to the average. Normal state is the better state.

That a subsystem of the steady multilateral system is not running properly (or disease, abnormal) is that the energy deviation from the average of the subsystems is too large, the high (real disease) or the low (virtual disease).

In addition to study these real or virtual diseases, TCE is also often considered a kind of relation diseases. The relation disease is defined as the relation of two sick subsystems. In general, a relation disease is less serious if the relation satisfies one of both the loving relationship and the killing relationship of the steady multilateral system. In this case, in general, the PPI inflation rate $x \in[0.007362,0.064920]$ which means $\rho_{0} \leq \rho_{1}=\rho(x) \leq 1$. This relation disease is less serious because this relation disease can not undermine the loving order or the killing order of the steady multilateral system. The less serious relation disease can make the intervention increasing the sizes of both the intervention reaction coefficients $\rho_{1}, \rho_{2}$ and the self-protection coefficient $\rho_{3}$.

But the relation disease is more serious if the relation not only doesn't satisfy the killing relationship of the steady multilateral system, but also can destroy the killing order, i.e., there is an incest order. In this case, in general, the PPI inflation rate $x \notin[0.007362,0.064920]$ which means 
$0<\rho_{1}=\rho(x)<\rho_{0}$. This relation disease is more serious because the relation disease can destroy the killing order of the steady multilateral system if the disease continues to develop. The more serious relation disease can make both the intervention reaction coefficients $\rho_{1}, \rho_{2}$ and the selfprotection coefficient $\rho_{3}$ decreasing response to intervention.

There are also many relation diseases which cannot destroy the loving order or the killing order of the steady multilateral system although the relation doesn't satisfy strictly the loving relationship or the killing relationship of the steady multilateral system, i.e., there is not an incest order. These relation diseases are called rare since they are hardly occurrence for a healthy economy.

The purpose of intervention is to make the steady multilateral system return to normal state. The method of intervention is to increase or decrease the energy of a subsystem.

What kind of intervening should follow the principle to treat it? Western mathematics emphasizes directly mathematical treatments on disease subsystem after the disease of subsystem has occurred, but the indirect intervening of oriental mathematics of TCE is required before the disease of subsystem will occur. In mathematics, which is more reasonable?

Based on this idea, many issues are worth further discussion. For example, if an intervening has been implemented to a disease subsystem before the disease of subsystem will occur, what relation disease will be less serious which does not need to be intervened? what relation disease will be more serious which needs to be intervened?

\subsection{Kinds of Relation Disease of Steady Multilateral Systems}

For a steady multilateral system $V$ with two incompatibility relations, suppose that the subsystems $X, X_{S}, X_{K}, K_{X}, S_{X}$ are the same as those defined in Theorem 3.1. Then the relation diseases can be decomposed into the following classes:

Definition 3.1 (involving (相及) and infringing (相犯) ) Suppose that both $x$ and $X_{S}$ having the loving relationship fall ill. Consider a relation disease occurred between $x$ and $X_{S}$.

The relation disease between $x$ and $X_{S}$ is called less serious if $x$ is a virtual disease and so is $X_{S}$ at the same time. The less serious relation disease between virtual $x$ and virtual $X_{S}$ is also called a mother's disease involving in her son. The mother is the cause of disease.

The relation disease between $x$ and $X_{S}$ is also called less serious if $x$ is a real disease and so is $X_{S}$ at the same time. The less serious relation disease between real $X$ and real $X_{S}$ is also called a son's disease infringing upon its mother. The son is the cause of disease.

The relation disease between $x$ and $x_{S}$ is called rare if $x$ is a real disease but $x_{S}$ is a virtual disease at the same time, or if $x$ is a virtual disease but $x_{S}$ is a real disease at the same time. The rare relation disease implies that they cannot destroy the loving order although real $x$ cannot love virtual $X_{S}$ or virtual $x$ cannot love real $X_{S}: \#$ 
Definition 3.2 (multiplying (相乘) and insulting (相侮)) Suppose that both $x$ and $X_{K}$ having the killing relationship fall ill. Consider a relation disease occurred between $x$ and $X_{K}$. The relation disease between $x$ and $x_{K}$ is called less serious if $x$ is a real disease and $x_{K}$ is a virtual disease at the same time. The less serious relation disease between $x$ and $x_{K}$ is also called a multiplying relation.

The relation disease between $x$ and $x_{K}$ is called more serious if $x$ is a virtual disease but $x_{K}$ is a real disease at the same time. The more serious relation disease between $x$ and $X_{K}$ is also called an insulting relation. It means that $x$ has been harmed by $x_{K}$ through the method of incest.

The relation disease between $x$ and $X_{K}$ is called rare if $x$ is a real disease and so is $X_{K}$ at the same time, or if $x$ is a virtual disease and so is $X_{k}$ at the same time. The rare relation disease implies that they cannot destroy the killing order from $x$ to $X_{K}$ although real $x$ cannot kill real $X_{K}$ or virtual $x$ cannot kill virtual $X_{K}$.

The relation disease between $x, X_{K}$ and $K_{X}$ is called more serious if $x$ is a real disease, and $X_{K}$ is a virtual disease but $K_{X}$ is also a virtual disease at same time, i.e., not only real $x$ multiplies in virtual $X_{K}$, but also insults virtual $K_{X}$ by using the method of incest. It is because the energy of real $x$ is too high. The more serious relation disease between $x, X_{k}$, and $K_{X}$ is also called a multiplying-insulting (乘侮) relation.

The relation disease between $x, X_{K}$, and $s_{X}$ is called more serious if $x$ is a real disease, and $X_{K}$ is a virtual disease but $s_{X}$ is also real disease at same time, i.e., not only real $x$ multiplies in virtual $X_{K}$, but also real $s_{X}$ insults virtual $X_{K}$ by using the method of incest. It is because the energy of virtual $X_{K}$ is too low. The more serious relation disease between $X, X_{K}$, and $s_{X}$ is also called a multiplying-insulting relation.

Only the more serious relation disease can destroy the killing relationship order of the Yin Yang Wu Xing system. All the therapeutic principles need to prevent the more serious relation disease occurrence in the first place.\#

The disease of multiplying-insulting relation will result in more than three subsystems fallingill. Generally, three or more subsystems falling-ill, it will be difficult to treat. Therefore, the multiplying-insulting relation disease should be avoided as much as possible. In Chinese words, it is that"Again and again, not only to the repeated to four” (只有再一再二, 没有再三再四) Allow one or two subsystems fall ill, but Don't allow three or four subsystems fall ill.

\subsection{First Transfer Laws of Diseases of Steady Multilateral Systems with a healthy Economy}

Suppose that a steady multilateral system ${ }_{v}$ having energy function $\varphi\left(^{*}\right)$ is normal or healthy. Let $x$ be the PPI inflation rate of $v$. Taking $\rho_{1}=\rho(x), \rho_{2}=\rho(x)^{2}$, and $\rho_{3}=c \rho(x)$ where $0 \leq c \leq 1$ and $\rho(x)$ is defined in Eqs.(3) and (4). The healthy economy means that the conditions $\rho_{0} \leq \rho(x) \leq 1$ and $0<c \leq 1$ hold, which is equivalent to the normal range $x \in[0.007362,0.064920]$ or the healthy condition $\rho_{1}+\rho_{2} \rho_{3} \geq 1-\rho_{2} \rho_{3}$. That $c \rightarrow 0$ implies that the body is without the ability of 
self-protection, i.e., $\rho_{3}=c \rho(x) \rightarrow 0$. Of course, the body cannot be healthy. It is because for any $x \neq 3.1359 \%$, when $c \rightarrow 0$, there are

$$
\rho_{1}+\rho_{2} \rho_{3}=\rho(x)+c \rho(x)^{3} \rightarrow \rho(x)<1 \leftarrow 1-c \rho(x)^{3}=1-\rho_{2} \rho_{3},
$$

such that the healthy condition $\rho_{1}+\rho_{2} \rho_{3} \geq 1-\rho_{2} \rho_{3}$ cannot hold.

By using Corollary 2.1 and Theorems 2.1 and 3.1, the following Theorem 3.2 can be obtained as the transfer law of occurrence and change of diseases with a healthy economy.

\section{Theorem 3.2 Let the PPI inflation rate $x \in[0.007362,0.064920]$ which is equivalent to the conditions $\rho_{0} \leq \rho_{1}=\rho(x) \leq 1$ and $0<c \leq 1$.}

In this case, almost always, the less serious relation disease will occur and change. If the disease continues to develop, the change can make a more serious relation disease occur.

The occurrence and change of diseases with a healthy economy has its transfer law: The first occurrence and change of the loving relationship and the killing relationship after the loving relationship disease. In other words, the following statements are true.

(1).If a subsystem $x$ of a steady multilateral system $v$ falls a virtual disease, the transfer law is the first occurrence of the virtual disease of the mother $s_{x}$ of $x$ with a less serious relation disease between virtual $s_{x}$ and virtual $x$, and secondly the real disease of the bane $K_{X}$ of $x$ after the virtual disease of $S_{X}$ with a less serious relation disease between real $K_{X}$ and virtual $x$, and thirdly the real disease of the prisoner $X_{K}$ of $x$ with a more serious relation disease between real $X_{K}$ and virtual $x$, and fourthly the virtual disease of the son $X_{S}$ of $x$ with a less serious relation disease between virtual $X_{S}$ and virtual $x$, and finally the new remission virtual disease of the subsystem $x$ itself, and for the next round of disease transmission, until disease rehabilitation.

(2).If a subsystem $x$ of a steady multilateral system $v$ encounters a real disease, the transfer law is the first occurrence of the real disease of the son $X_{S}$ of $x$ with a less serious relation disease between real $X_{S}$ and real $x$, and secondly the virtual disease of the prisoner $X_{K}$ of $x$ after the real disease of $X_{S}$ with a less serious relation disease between virtual $X_{K}$ and real $x$, and thirdly the virtual disease of the bane $K_{x}$ of $x$ with a more serious relation disease between virtual $K_{X}$ and real $x$, and fourthly the real disease of the mother $s_{X}$ of $x$ with a less serious relation disease between real $s_{X}$ and real $X$, and finally the new abated real disease of the subsystem $X$ itself, and for the next round of disease transmission, until disease rehabilitation.

All first transfer laws of diseases with a healthy complex system are summed up as Figures 2 and 3.\# 


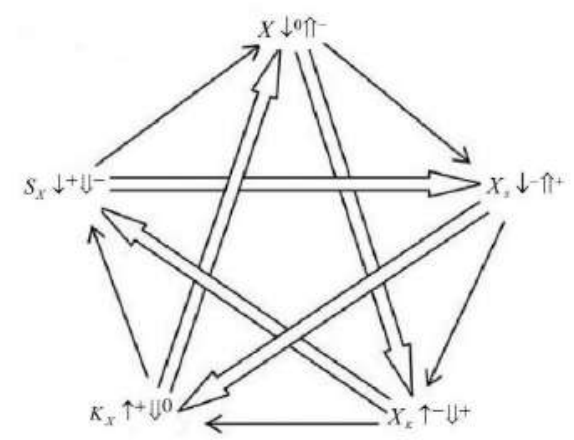

Figure 2. Transfer law of virtual diseases for a healthy economy

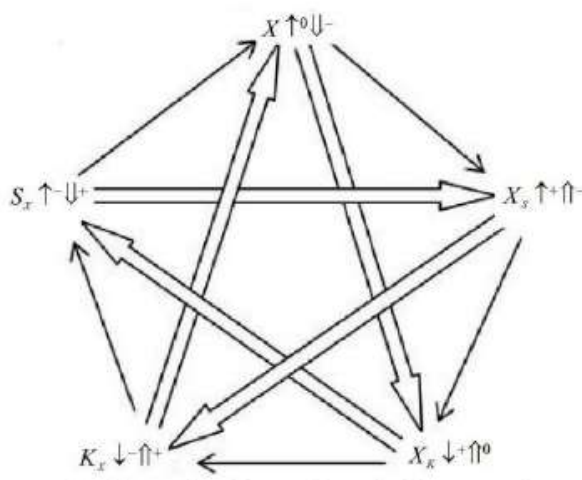

Figure 3. Transfer law of real diseases for a healthy economy

Remark 4. Theorem 3.2 is called the transfer law of occurrence and change of diseases with a healthy economy, simply, the first transfer law. For a real disease, the first transfer law is along the loving relationship order transmission as follows:

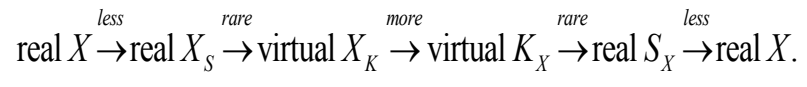

For a virtual disease, the first transfer law is against the loving relationship order transmission as follows:

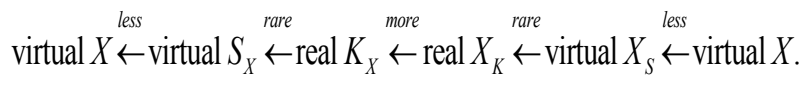

The transfer relation of the first transfer law running is the loving relationship, denoted by $\rightarrow$. The running condition of the first transfer law is both $\left(\rho_{1}+\rho_{2} \rho_{3}\right) \geq\left(1-\rho_{2} \rho_{3}\right)$ and $\rho_{3}=c \rho(x)>0$. By Theorem 2.1 and Corollary 2.1, the running condition is nearly equivalent to both $\rho_{0} \leq \rho_{1}=\rho(x) \leq 1$ and $0<c \leq 1$. The best-state condition of the first transfer law is $\rho_{3}=c \rho(x)$ where $c \rightarrow 1$ which is the best state of $\rho_{3}$ for a healthy economy. To follow or utilize the running of the first transfer law is equivalent to the following method. For dong so, it is in order to protect or maintain the loving relationship. The method can strengthen both the value $\left(\rho_{1}+\rho_{2} \rho_{3}\right)=\left(\rho(x)+c \rho(x)^{3}\right)$ tending to be large and the value $\left(1-\rho_{2} \rho_{3}\right)=\left(1-c \rho(x)^{3}\right)$ tending to be small at the same time. In other words, the way can make all of both $\rho(x)$ and $c$ tending to be large. It is because the running condition of the loving relationship $\left(\rho_{1}+\rho_{2} \rho_{3}\right) \geq\left(1-\rho_{2} \rho_{3}\right)$ is the stronger the use, which dues to $\rho_{1}=\rho(x)$ the greater the use. In other words again, if the treatment principle of the loving relationship disease is to use continuously abiding by the first transfer law, then all of both the intervention reaction coefficients $\rho_{1}=\rho(x), \rho_{2}=\rho(x)^{2}$ and the coefficient of self-protection $\rho_{3}=c \rho(x)>0$ where $0<c \leq 1$ will tend to be the best state, i.e., $\rho(x) \rightarrow 1$ and $0<c \rightarrow 1$. 
Side effects of treating problems were the question: in the treating process, destroyed the balance of the normal subsystems which are not sick or intervened systems. The energy change of the intervened system is not the true side effects issue. The energy change is called the pseudo or non-true side effects issue since it is just the food of the second physiological system of the steady multilateral system for a healthy economy. The best state of the selfprotection coefficient, $\rho_{3}=c \rho(x) \rightarrow \rho(x)=\rho_{1}$, where $c \rightarrow 1$, implies the non-existence of any side effects issue if the treatment principle of TCE is used. Therefore any disease that causes side effects issue occurrence in the first place dues to the non-best state of selfprotection ability, i.e., $\rho_{3}=c \rho(x)<\rho(x)=\rho_{1}$. To follow or utilize the running of the first transfer law can make both $\rho(x) \rightarrow 1$ and $0<c \rightarrow 1$. At this point, the paper advocates to follow or utilize the first transfer law. It is in order to avoid the side effects issue occurrence for the healthy economy. \#

\subsection{Second Transfer Laws of Diseases of Steady Multilateral Systems with an unhealthy Economy}

Suppose that a steady multilateral system $v$ having energy function $\varphi(*)$ is abnormal or unhealthy. Let $x$ be the PPI inflation rate of $v$. Taking $\rho_{1}=\rho(x), \rho_{2}=\rho(x)^{2}$ and $\rho_{3}=c \rho(x)$ where $0 \leq c \leq 1$, and $\rho(x)$ is defined in Eqs.(3) and (4). The unhealthy economy means that the conditions $\rho_{0}>\rho_{1}=\rho(x)>0$ and $0 \leq c \leq 1$ hold, which is equivalent to the abnormal range $x \notin[0.007362,0.064920]$.

From [20] and by using Corollary 2.1 and Theorems 2.1 and 3.1, the following Theorem 3.3 can be obtained as the transfer law of occurrence and change of diseases with an unhealthy economy.

\section{Theorem 3.3 Let the PPI inflation rate $x \notin[0.007362,0.064920]$ which is equivalent to the conditions $\rho_{0}>\rho_{1}=\rho(x)>0$ and $0 \leq c \leq 1$.}

In this case, almost always, the rare relation disease will occur and change. If the disease continues to develop, the change can make a more serious relation disease occur.

The transfer of disease with an unhealthy economy has its transfer law: Only there is the transfer of the killing relationship. In other words, the following statements are true.

(1).If a subsystem $x$ of a steady multilateral system $V$ falls a virtual disease, then the disease comes from the son $X_{S}$ of $x$. The transfer law is the first occurrence of the virtual disease of the prisoner $X_{K}$ of $x$ with a rare relation disease between virtual $x$ and virtual $X_{K}$, and secondly the virtual disease of the mother $s_{X}$ of $x$ after the virtual disease of $X_{K}$ with a rare relation disease between virtual $X_{K}$ and virtual $s_{X}$, and thirdly the virtual disease of the son $X_{S}$ of $x$ with a rare relation disease between virtual $S_{X}$ and virtual $X_{S}$, and fourthly the real disease of the bane $K_{X}$ of $X$ with a more serious relation disease between virtual $X_{S}$ and real $K_{X}$, and finally the new remission virtual disease of the subsystem $x$ itself with a less serious relation disease between real $_{K_{X}}$ and virtual $X$, and for the next round of disease transmission, until disease rehabilitation.

(2).If a subsystem $x$ of a steady multilateral system $v$ falls a real disease, then the disease comes from the mother $s_{x}$ of $x$. The transfer law is the first occurrence of the real disease of the 
bane $K_{X}$ of $x$ with a rare relation disease between real $x$ and real $K_{X}$, and secondly the real disease of the son $X_{S}$ of $x$ after the real disease of the bane $K_{x}$ of $x$ with a rare relation disease between real $K_{X}$ and real $X_{S}$, and thirdly the real disease of the mother $s_{X}$ of $x$ with a rare relation disease between real $X_{S}$ and real $s_{X}$, and fourthly the virtual disease of the prisoner $X_{K}$ of $x$ with a more serious relation disease between real $_{X} S_{X}$ and virtual $X_{K}$, and finally the new abated real disease of the subsystem $X$ itself with a less serious relation disease between virtual $X_{K}$ and real $X$, and for the next round of disease transmission, until disease rehabilitation.

All second transfer laws of diseases with an unhealthy economy are summed up as Figures 4 and 5.\#

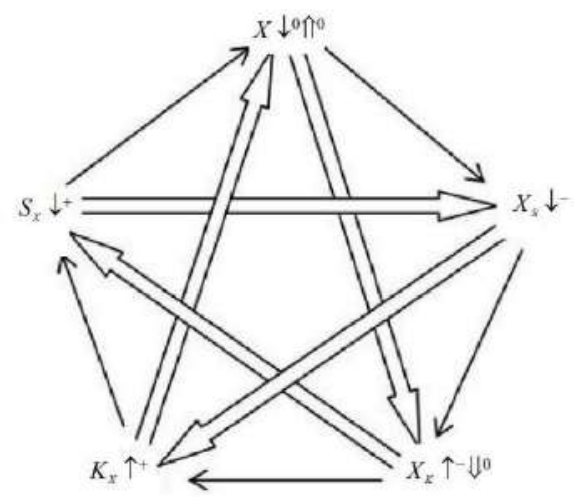

Figure 4. Transfer law of virtual diseases for an unhealthy economy

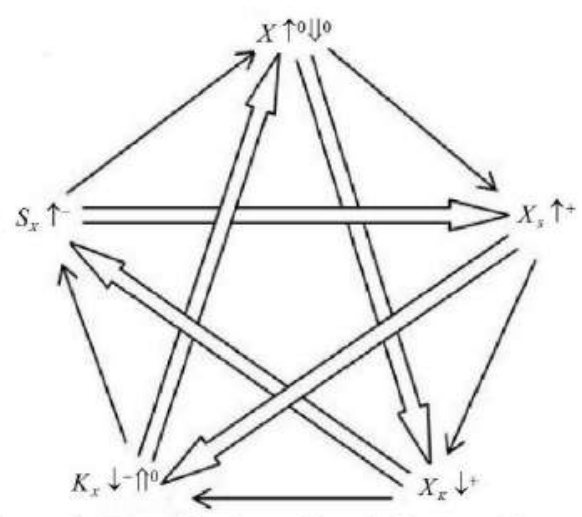

Figure 5. Transfer law of real diseases for an unhealthy economy

Remark 5. Transfer law of Theorem 3.3 is called transfer law of occurrence and change of diseases with an unhealthy economy, simply, the second transfer law. For a virtual disease, the second transfer law is along the killing relationship order transmission as follows:

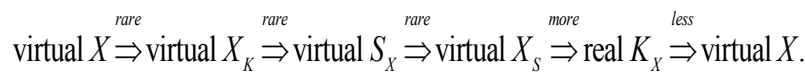

For a real disease, the second transfer law is against the killing relationship order transmission as follows:

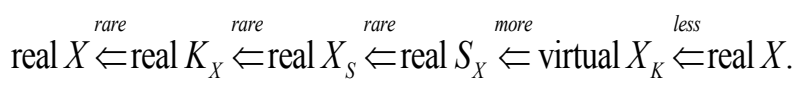

The transfer relationship of the second transfer law running is the killing relationship, denoted by $\Rightarrow$. The running condition of the second transfer law is both 


$$
\left(\rho_{1}+\rho_{2} \rho_{3}\right)<\left(1-\rho_{2} \rho_{3}\right) \text { and } \rho_{3}=c \rho(x) \geq 0 .
$$

By Theorem 2.1 and Corollary 2.1, the running condition is equivalent to both $\rho_{0}>\rho_{1}=\rho(x)>0$ and $1 \geq c \geq 0$. That $\rho_{3}=c \rho(x) \rightarrow 0$ means the lack of capability of self-protection. Of course, it is the basis condition of running the second transfer law.

The stopping condition of the second transfer law is both $\left(\rho_{1}+\rho_{2} \rho_{3}\right) \geq\left(1-\rho_{2} \rho_{3}\right)$ and $\rho_{3}=c \rho(x)>0$, which is the running condition of the first transfer law, or, the existence condition of capabilities of both intervention reaction and self-protection. To follow or utilize the running of the second transfer law is equivalent to the following method. For dong so, it is to protect and maintain the killing relationship of the steady multilateral system. The method can strengthen all of both $\rho_{1}-\rho_{3}=\rho(x)(1-c)$ and $\rho_{2}-\rho_{1} \rho_{3}=\rho(x)^{2}(1-c)$ tending to be small at the same time. In other words, using the method can make $c$ tends to be large for a fixed $\rho(x)>0$.It is because the transferring condition of the killing relation disease $\left(\rho_{1}+\rho_{2} \rho_{3}\right)<\left(1-\rho_{2} \rho_{3}\right)$ is the weaker the use, which dues to $\rho_{3}=c \rho(x)$ is the greater the use. The transferring way can make both $\rho_{1}-\rho_{3} \rightarrow 0$ and $\rho_{2}-\rho_{1} \rho_{3} \rightarrow 0$ at the same time such that the killing relation disease cannot be transferred. In other words again, if the treatment principle of the killing relationship diseases is to use continuously abiding by the second transfer law, then the coefficient of self-protection will tend to be the occurrence state, i.e., $\rho_{3}=c \rho(x)>0$ where $1 \geq c \geq \frac{1-\rho(x)}{2 \rho(x)^{3}} \geq 0$, and the coefficients of intervention reaction also will tend to the healthy state, i.e., $\rho_{0} \leq \rho_{1}=\rho(x) \leq 1$ , such that $\left(\rho_{1}+\rho_{2} \rho_{3}\right) \geq\left(1-\rho_{2} \rho_{3}\right)$.\#

Medical and drug resistance problem is that such a question: beginning more appropriate medical treatment, but is no longer valid after a period. In the state

$\rho_{1}-\rho_{3}=\rho(x)(1-c) \rightarrow 0, \quad \rho_{2}-\rho_{1} \rho_{3}=\rho(x)^{2}(1-c) \rightarrow 0$, by Theorem 3.2, any medical and drug resistance problem is non-existence if the treatment principle of TCE is used. But in the state

$$
\rho_{1}-\rho_{3}=\rho(x)(1-c) \rightarrow \rho(x), \quad \rho_{2}-\rho_{1} \rho_{3}=\rho(x)^{2}(1-c) \rightarrow \rho(x)^{2},
$$

by Theorem 3.3, the medical and drug resistance problem is always existence, even if the treatment principle of TCE has been used. In other words, the lack of capability of selfprotection, i.e., $\rho_{3}=c \rho(x) \rightarrow 0$, implies the possible existence of a medical and drug resistance problem, although the treatment principle of TCE has been used. At this point, the paper advocates to follow or utilize the second transfer law. It is in order to prevent and avoid the medical and drug resistance problem occurrence for the unhealthy economy.\#

\section{TREATMENT PRINCIPLE OF TCE}

In order to explain treatment principle of TCE, the changes in the PPI inflation rate range can be divided into four parts. From [20] , Corollary 2.1 and Theorems 2.1, 3.2 and 3.3, it can be easily proved that the following theorem is true.

Theorem 4.1 Suppose that the subsystem $x$ of a steady multilateral system falls ill. Let $x$ be the PPI inflation rate of the steady multilateral system. Denoted the parameters of the normal range as follows 


$$
a=0.7362 \%, b=6.4920 \%, t_{0}=3.1359 \%
$$

Then the following statements are true.

1) Suppose that $x<a$ as virtual, in which $x$ falls a virtual disease with an unhealthy economy. The primary treatment is to increase the energy of the subsystem $x$ directly. And the secondary treatment is to increase the energy of the $\operatorname{son} X_{S}$ of $x$, and at the same time, to decrease the energy of the prisoner $K_{X}$ of $X_{S}$.

2) Suppose that $x \in\left[a, t_{0}\right.$ ) as virtual-normal, in which $x$ will fall a virtual disease with a healthy economy. The primary treatment is to increase the energy of the mother subsystem $s_{x}$ of $x$ which is an indirect treating for $x$. And the secondary treatment is to increase the energy of $x$ itself, and at the same time, to decrease the energy of the prisoner $X_{K}$ of $X$.

3) Suppose that $x \in\left[t_{0}, b\right]$ as real-normal, in which $x$ will encounter a real disease with a healthy economy. The primary treatment is to decrease the energy of the son subsystem $X_{S}$ of $x$ which is an indirect treating for $x$. And the secondary treatment is to decrease the energy of $x$ itself, and at the same time, to increase the energy of the bane $K_{x}$ of $x$.

4) Suppose that $x>b$ as real, in which $x$ encounters a real disease with an unhealthy economy. The primary treatment is to decrease the energy of the subsystem $x$ directly. And the secondary treatment is to decrease the energy of the mother $s_{x}$ of $x$, and at the same time, to increase the energy of the bane $X_{K}$ of $s_{X}$. \#

Remark 6. Treatment principle of Theorem 4.1 based on ranges of the PPI inflation rate is called the treatment principle of TCE, since it is in order to protect and maintain the balance of two incompatibility relations: the loving relationship and the killing relationship.

For the unhealthy economy where $x<a$ or $x>b$, the treatment principle is the method for doing so in the following:

The primary treatment is to increase or decrease the energy of $x$ directly corresponding to $x<a$ or $x>b$ respectively, and the secondary treatment is to increase the energy of $X_{S}$ or $X_{K}$ while to decrease the energy of $K_{X}$ or $s_{X}$, respectively.\#

The primary treatment is in order to protect and maintain the loving relationship, abiding by TCE's ideas "Virtual disease with an unhealthy economy is to fill itself" and "Real disease with an unhealthy economy is to rush down itself". It is because the method for dong so is not only greatly medical diseases of their own, but also provides the pseudo side effects as the food for the second physiological system. The method is to promote the first physiological system running since the second physiological system controls the first physiological system. And it is also to improve the loving relationship to develop since the loving relationship mainly comes from the first physiological system. The loving relationship to develop can strengthen both that $\rho_{1}+\rho_{2} \rho_{3}=\rho(x)+c \rho(x)^{2}$ tends to be large and that $1-\rho_{2} \rho_{3}=1-c \rho(x)^{3}$ tends to be small at the same time. In other words, the way can make all of both $\rho(x)$ and $c$ tend to be large, at least, $c$ greater than zero for an unhealthy economy and $\rho_{0} \leq \rho(x) \leq 1$, such that the economy from unhealthy to healthy, or the first physiological system works, or, the occurrence of capability of self-protection, or, the running of the first transfer law, or, the stopping of the second transfer law. 
The secondary treatment is in order to protect or maintain the killing relationship, abiding by TCE's ideas "Don't have disease cure cure non-ill" and "Strong inhibition of the same time, support the weak". By the second transfer law in Theorem 3.3, the more serious relation disease is the relation disease between virtual $X_{S}$ and real $K_{X}$, or between real $s_{X}$ and virtual $x_{K}$ respectively.

Abiding by TCE's idea 'Strong inhibition of the same time, support the weak", it must be done to increase the energy of $X_{S}$ or $X_{K}$ while decrease the energy of $K_{X}$ or $S_{X}$ respectively.

Abiding by TCE's idea "Don't have disease cure cure non-ill", it must be done to prevent or avoid the more serious relation disease between virtual $x_{S}$ and real $K_{K_{X}}$ or between real $s_{x}$ and virtual $X_{K}$ occurrence respectively.

The method for doing so can improve the killing relationship to develop since real $x_{S}$ or $X_{K}$ can kill virtual $K_{X}$ or $s_{x}$ respectively. The killing relationship to develop means that both $\rho_{1}-\rho_{3}=\rho(x)(1-c)$ and $\rho_{2}-\rho_{1} \rho_{3}=\rho(x)^{2}(1-c)$ tend to be small at the same time. In other words, the way can make, for fixed $\rho(x), c$ tending to be large, at least, greater than zero for an unhealthy economy, such that the economy from unhealthy to healthy, or the first physiological system works, or, the occurrence of capability of self-protection, or, the running of the first transfer law, or, the stopping of the second transfer law.

For the healthy economy where $x \in\left[a, t_{0}\right)$ or $x \in\left[t_{0}, b\right]$, the treatment principle is the method for doing so in the following:

The primary treatment is to increase or decrease the energy of $s_{x}$ or $x_{S}$ corresponding to $x \in\left(a, t_{0}\right]$ or $x \in\left[t_{0}, b\right]$ respectively, and the secondary treatment to increase the energy of $K_{x}$ or $x$ while to decrease the energy of $x$ or $X_{k^{\prime}}$ respectively.\#

The primary treatment is in order to protect and maintain the loving relationship, abiding by TCE's ideas "Virtual disease with a healthy economy is to fill mother" and "Real disease with a healthy economy is to rush down its son". It is because the method for dong so is not only greatly medical diseases of their own, but also provides the pseudo side effects as the food for the second physiological system. The method is to promote the first physiological system running since the second physiological system controls the first physiological system. And it is also to improve the loving relationship developing since the loving relationship mainly comes from the first physiological system. The loving relationship developing can strengthen both that $\rho_{1}+\rho_{2} \rho_{3}=\rho(x)+c \rho(x)^{2}$ tends to be large and that $1-\rho_{2} \rho_{3}=1-c \rho(x)$ tends to be small at the same time. In other words, using the way can make all of both $\rho(x)$ and $0<c$ tending to be large, the best, all equal to 1 for a healthy economy, such that the capability of self-protection is in the best state, or, the non-existence of side effects issue, or, the non-existence of medical and drug resistance problem.

The secondary treatment is in order to protect or maintain the killing relationship, abiding by TCE's ideas "Don't have disease cure cure non-ill" and "Strong inhibition of the same time, support the weak". By the first transfer law, the more serious relation disease is the relation disease between real $x$ and virtual $K_{x}$ or between virtual $x$ and real $X_{K}$ corresponding to real $x$ or virtual $x$, respectively. 
Abiding by TCE's idea "Don't have disease cure cure non-ill”, it must be done to prevent and avoid the more serious relation disease between real $x$ and virtual $K_{x}$ or between virtual $x$ and real $X_{K}$ occurrence corresponding to real $x$ or virtual $x$ respectively.

Abiding by TCE's idea "Strong inhibition of the same time, support the weak", it must be done to increase the energy of $K_{x}$ or $x$ while decrease the energy of $x$ or $X_{K}$ respectively.

The method for doing so can improve the killing relationship developing since real $K_{X}$ or real $X$ can kill virtual $x$ or virtual $X_{K}$ respectively. The killing relationship developing also means that both $\rho_{1}-\rho_{3}=\rho(x)(1-c)$ and $\rho_{2}-\rho_{1} \rho_{3}=\rho(x)^{2}(1-c)$ tend to be small at the same time. In other words, using the way can make, for fixed $\rho(x), 0<c$ tending to be large, the best, equal to 1 for a healthy economy, such that the capability of self-protection is in the best state, or, the non-existence of side effects, or, the non-existence of medical and drug resistance issue. \#

\section{CHINESE PPI FOR THE WOOD SUBSYSTEM}

Suppose that $M_{2}$ as issued in the circulation of money and GDP as Gross Domestic Product in Chinese from 1990 to 2014, the annual PPI and the annual PPI inflation rates can be measured in Table 1.

Also watching Table 1, the state of the PPI inflation rate is: virtual-normal, real-normal, real, real, real, real-normal, for 1991-1996, respectively.During this period of time, large-scale goods have been made.

It means that the subsystem $\operatorname{wood}(x)$ of the economic social system with an unhealthy economy encounters a real economic disease since the PPI inflation rate belongs to "industry" of $\operatorname{wood}(x)$.

So, at present the most serious problem is to treat the $\operatorname{subsystem} \operatorname{wood}(x)$ falling a real disease for an unhealthy sub-economy. It is the case in (4) of Theorem 4.1 for $\operatorname{wood}(x)$.

By (4) of Theorem 4.1 again, the primary treatment is to decrease the energy of the subsystem $\operatorname{wood}(x)$ directly. And the secondary treatment is to decrease the energy of the mother water( $\left.S_{X}\right)$ of $\operatorname{wood}(X)$, and at the same time, to increase the energy of the bane earth $\left(X_{K}\right)$ of water( $\left.S_{X}\right)$.

In fact, the Chinese government did just that. For 1993-1999, not only had decreased gradually the financial amounts of investment in the manufacture (to decrease the energy of the subsystem $\operatorname{wood}(x)$ directly), but also had decreased investment in the Army, such as, big disarmament, a freeze on a large number of military engineering, etc. (for decreasing the energy of water $\left(S_{x}\right)$ ) while had increased little by little the workers' wages, the social security and social welfare, such as, the public accumulation fund for housing construction, pension funds, medical insurance, unemployment insurance, etc. (to increase the energy of earth $\left(x_{K}\right)$ ).

Also watching Table 1, the state of the PPI inflation rate is virtual-normal, virtual, virtual, virtual, virtual, virtual, for 1997-2003, respectively.During this period of time, mass goods cannot be made. Supplies are still scarce. Rush on still appear on the market. 
It means that the subsystem wood $(x)$ falls a virtual disease for an unhealthy economy since the PPI inflation rate belongs to the "industry" of $\operatorname{wood}(x)$.

Also watching Table 1, the state of the CPI inflation rate is: virtual-normal, virtual, virtual, virtual, virtual, virtual, virtual, for 1997-2003, respectively.

It means that the subsystem earth $\left(x_{K}\right)$ of the economic social system with an unhealthy economy encounters a virtual economic disease since the manufacture of large-scale goods or the CPI inflation rate belongs to"industry" of earth $\left(X_{K}\right)$.

There are two subsystems $\operatorname{wood}(x)$ and earth $\left(x_{K}\right)$ which have the killing relation and falling virtual diseases. By Definition 3.2, the relation disease between virtual $\operatorname{wood}(x)$ and virtual earth $\left(x_{K}\right)$ is rare since virtual wood $(x)$ cannot kill virtual earth $\left(x_{K}\right)$ which cannot destroy the killing order from $\operatorname{wood}(x)$ to earth $\left(x_{K}\right)$. But if the subsystem earth $\left(x_{K}\right)$ is intervened such that it is from virtual to real, there is a more serious relation disease between virtual wood $(x)$ and real earth $\left(x_{K}\right)$. It is because the virtual $\operatorname{wood}(x) \operatorname{cannot}$ kill the real earth $\left(x_{K}\right)$ which can destroy the balance of the killing relation from $\operatorname{wood}(x)$ to earth $\left(x_{K}\right)$.

So, at present the most serious problem is to treat the subsystem $\operatorname{wood}(x)$ falling a virtual disease for an unhealthy sub-economy. It is the case in (1) of Theorem 4.1 for $\operatorname{wood}(x)$.

By (1) of Theorem 4.1, the primary treatment is gotten to increase the energy of the subsystem $\operatorname{wood}(x)$ directly. And the secondary treatment is gotten to increase the energy of the son fire( $\left.x_{S}\right)$ of $\operatorname{wood}(x)$, and at the same time, to decrease the energy of the prisoner metal $\left(K_{X}\right)$ of fire( $\left.X_{S}\right)$.

In fact, the Chinese government did just that. For 1999-2008, not only had increased gradually the financial amounts of investment in the manufacture (e.g.,to invest in real estate, to increase the energy of the subsystem $\operatorname{wood}(x)$ directly), but also had increased to make money, and investment in the agriculture, such as, exempt from the agricultural taxation, increase of agricultural land expropriation compensation, etc. (to increase the energy of fire $\left(x_{S}\right)$ including jun-fire $\left(x_{S}^{j}\right)$ and xiang-fire $\left.\left(x_{S}^{x}\right)\right)$ while had decreased in the science and education, such as, a small amount of teachers and researchers for a raise, schools and research institutions selfsustaining, etc.(to decrease the energy of $\operatorname{metal}\left(K_{X}\right)$ ).

Therefore, application of nature for the treatment principle of TCE by the Chinese government had brought the sustained and rapid growth of industry economy for 1991-2008.

Also watching Table 1 again, the state of the PPI inflation rate is: virtual-normal, virtual-normal, virtual-normal, real-normal, real-normal, virtual, virtual-normal, real-normal, for 2004-2011, respectively. During this period of time, large-scale goods have been still made. But a lot of society problems begin occurring.

It means the subsystem $\operatorname{wood}(x)$ is mainly with a healthy sub-economy. It is because the manufacture of large-scale goods or the normal PPI inflation rate belongs to "industry" of the subsystem $\operatorname{wood}(x)$. 
But the Finance inflation rate is continuously smaller than $a^{6}=2.9515 \%$ (deflation) from 2004 to 2008 as virtual, except for 2005 year's normal state. It means the whole economy still is unhealthy. Its some subsystem still has encountered a virtual disease. Observation of the whole economic and social five subsystems, it can be found that there is a virtual disease state of the subsystem earth $\left(X_{K}\right)$.

In fact, also watching Table 1 again, the state of the CPI inflation rate is: real-normal, virtual, virtual, real-normal, real, virtual, virtual-normal, real-normal, for 2004-2011, respectively.

It means the subsystem earth $\left(X_{K}\right)$ is mainly with an unhealthy sub-economy and falls an expected virtual disease. It is because the CPI inflation rate belongs to the "commerce" of $\operatorname{earth}\left(x_{K}\right)$.

There are two subsystems $\operatorname{wood}(x)$ and earth $\left(x_{K}\right)$ which have the killing relation. But the $\operatorname{subsystem} \operatorname{wood}(x)$ is real-normal and the subsystem earth $\left(x_{K}\right)$ falls a virtual disease. By Definition 3.2, the relation disease between real $\operatorname{wood}(x)$ and virtual earth $\left(x_{K}\right)$ is less since real $\operatorname{wood}(x)$ can kill virtual earth $\left(x_{K}\right)$ which cannot destroy the killing order from wood $(x)$ to earth $\left(x_{K}\right)$. Now the subsystem earth $\left(x_{K}\right)$ can be intervened such that it is from virtual to realnormal.

So, at present the most serious problem is to treat the subsystem earth $\left(x_{K}\right)$ falling a virtual disease. It is the case in (1) of Theorem $\mathbf{4 . 1}$ for earth $\left(x_{K}\right)$.

The $x_{K}$ as $x$ in theorem 4.1, using (1) of Theorem 4.1 again, the primary treatment is gotten to increase the energy of the subsystem earth $\left(X_{K}\right)$ directly. And the secondary treatment is gotten to increase the energy of the son metal $\left(K_{X}\right)$ of earth $\left(X_{K}\right)$, where $\left(X_{K}\right)_{S}=K_{X}$ in Figure 1, and at the same time, to decrease the energy of the bane $\operatorname{wood}(X)$ of earth $\left(X_{K}\right)$, where $K_{\left(X_{K}\right)}=X$ in Figure 1. In fact, the Chinese government did just that. For 2004-2014, not only had increased the financial amounts of investment in commerce, such as, strengthen the support for the WTO trade, etc. (to increase the energy of the subsystem earth $\left(x_{K}\right)$ directly), but also had increased investment in science, education and public facilities, such as to build high speed rail, etc. (to increase the energy of metal $\left(K_{X}\right)$ ) while had reduced the industrial support, such as, the appreciation of the RMB, etc. (to decrease the energy of $\operatorname{wood}(x)$ ).

Therefore, again application of nature for the treatment principle of TCE by the Chinese government had brought the 2004-2014 economic taking off again.

Also watching Table 1 again and again, the state of the PPI inflation rate is: virtual-normal, virtual-normal, virtual-normal, for 2012-2014, respectively.

It means that the the subsystem $\operatorname{wood}(x)$ is an expected virtual disease for a healthy subeconomy. It is because the manufacture of large-scale goods or the PPI inflation rate belongs to the "industry" of the subsystem $\operatorname{wood}(x)$.

The virtual-normal disease of $\operatorname{wood}(x)$ is not because of its low energy, but because of its energy is too high to make producing products too much, so much so that there is no way to sell products, low profit of industrial production. In the TCE, this disease is Yang irritability turned to deficiency disease. This disease is not the current urgent problems since it cannot 
destroy the killing order balance of the economy. But if this virtual-normal disease is continuously to develop, by Theorem 3.2, the virtual wood $(x)$ will make its mother subsystem water $\left(s_{X}\right)$ falling a virtual economic disease when it encounters an economic disease. In fact, the economic indicators of GDP which belongs to the subsystem water $\left(s_{X}\right)$ is beginning to decline. Abiding by TCE's idea "Don't have economic disease cure cure non-ill”, the prevention and treatment of the current work is the need to prevent the virtual disease of the subsystem water $\left(s_{X}\right)$ for a healthy economy.

So, at present the most serious problem is to treat the subsystem water $\left(s_{X}\right)$ falling a virtual disease with a healthy sub-economy of the subsystem water( $\left.s_{X}\right)$. It is the case in (2) of Theorem 4.1 for water $\left(s_{X}\right)$.

The $s_{X}$ as $x$ in (2) of Theorem 4.1, the primary treatment is gotten to increase the energy of the mother subsystem metal $\left(K_{X}\right)$ of the water $\left(s_{X}\right)$, where $S_{\left(S_{X}\right)}=K_{X}$ in Figure 1 . And the secondary treatment is gotten to increase the energy of the water $\left(s_{X}\right)$ itself while decrease the energy of the prisoner fire $\left(x_{S}\right)$ of the water $\left(s_{X}\right)$, where $\left(S_{X}\right)_{K}=X_{S}$ in Figure 1.

In fact, the Chinese government also is doing just that. Since 2015, not only has increased continuously investment in science, education and public facilities, such as, One Belt and One Road, etc. ( for increasing the energy of metal $\left(K_{X}\right)$ ), but also has increased to military spending (to increase the energy of the water $\left(s_{X}\right)$ ) while has reduced the number of making money, and reduced the agricultural support, such as, reduce the purchase price of agricultural products, etc. (to decrease the energy of fire $\left(x_{S}\right)$ including jun-fire $\left(x_{S}^{j}\right)$ and xiang-fire $\left(x_{S}^{x}\right)$ ). Therefore, again and again application of nature for the treatment principle of TCE by the Chinese government will lead to economic continue to glory since 2015.\#

\section{CONCLUSIONS}

This work shows how to treat the diseases of a economic society by using the PPI inflation rate $x$.For the PPI inflation rate, the normal range of theory is $[0.7362 \%, 6.4920 \%]$, nearly to $[1 \%, 6 \%]$, and the center is $3.1359 \%$, nearly to $3 \%$. The first or second transfer law of economic diseases changes according to the different economic society's PPI inflation rate whether in the normal range or not. For the normal range, the first transfer law in Theorem $\mathbf{3 . 2}$ runs; For the abnormal range, the second transfer law in Theorem $\mathbf{3 . 3}$ runs.

Assume that the range of economic society's PPI inflation rate $x$ is divided into four parts from small to large. Both second and third are for a healthy economy with a virtual or real disease respectively. The treating works are the prevention or treatment for a more serious relation disease between virtual $x$ and real $X_{K}$ or between virtual $K_{X}$ and real $x$ respectively. Each of both more serious relation diseases comes from the first transfer law in Theorem 3.2. And both first and fourth are for an unhealthy economy with a virtual or real disease respectively. The treating works are the prevention or treatment for a more serious relation disease between virtual $X_{S}$ and real $K_{X}$ or between virtual $X_{K}$ and real $S_{X}$ respectively. Each of both more serious relation diseases comes from the second transfer law in Theorem 3.3.

Economic disease treatment should protect and maintain the balance or order of two incompatibility relations: the loving relationship and the killing relationship. The method for 
doing so can make the $\rho_{3}=c \rho(x)$ tending to be large. In other words, using the method can make all of both $\rho(x)$ and $c$ tend to be large, at least, greater than zero for an unhealthy economy; or, the best, equal to 1 for a healthy economy.

The following way can make the capabilities of both intervention reaction and self-protection become in the best state, the non-existence of side effects issue, the non-existence of medical and drug resistance problem, and so on.

1) Suppose that $x<a=0.7362 \%$, in which $X$ falls a virtual disease with an unhealthy economy. In order to protect or maintain the loving relationship, abiding by TCE's idea “Virtual disease with an unhealthy economy is to fill itself" (虚则补之), increase the energy of $X$ directly.

In order to protect or maintain the killing relationship, abiding by TCE's idea "Don't have disease cure cure non-ill” (不治已病治未病), do a preventive treatment for the more serious relation disease between virtual $x_{S}$ and real $K_{X}$.

Through the intervening principle of "Strong inhibition of the same time, support the weak" (抑强扶弱), increase the energy of the son $X_{S}$ of $x$ while decrease the energy of the prisoner $K_{X}$ of $X_{S}$.

2) Suppose that $a=0.7362 \% \leq x<t_{0}=3.1359 \%$, in which $X$ will fall a virtual disease with a healthy economy. In order to protect and maintain the loving relationship, abide by TCE's idea "Virtual disease with a healthy economy is to fill its mother"(歔则补其母), increase the energy of the mother $s_{x}$ of $x$. The treating way is an indirect treating for $X$. In order to protect and maintain the killing relationship, abiding by TCE's idea“Don't have disease cure cure non-ill” (不治已病治未病), do a preventive treatment for the more serious relation disease between virtual $x$ and real $X_{K}$.

Through the intervening principle of "Strong inhibition of the same time, support the weak" (抑强扶弱), increase the energy of $x$ itself while decrease the energy of the prisoner $X_{K}$ of $x$.

3) Suppose that $t_{0}=3.1359 \% \leq x \leq b=6.4920 \%$, in which $x$ will encounter a real disease with a healthy economy. In order to protect or maintain the loving relationship, abiding by TCE's idea “Real disease with a healthy economy is to rush down its son" (实则泄其子), decrease the energy of the son $X_{S}$ of $x$. The treating way is an indirect treating for $x$. In order to protect or maintain the killing relationship, abiding by TCE's idea "Don't have disease cure cure non-ill" (不治已病治未病), do a preventive treatment for the more serious relation disease between real $x$ and virtual $K_{x}$.

Through the intervening principle of "Strong inhibition of the same time, support the weak" (抑强扶弱), decrease the energy of $x$ itself while increase the energy of the bane $K_{X}$ of $x$.

4) Suppose that $x>b=6.4920 \%$, in which $x$ encounters a real disease with an unhealthy economy. In order to protect or maintain the loving relationship, abiding by TCE's idea “Real disease with an unhealthy economy is to rush down itself” (实则泄之), decrease the energy of $x$ directly.

In order to protect or maintain the killing relationship, abiding by TCE's idea "Don't have disease cure cure non-ill” (不治已病治未病), do a preventive treatment for the more serious relation disease between real $S_{X}$ and virtual $X_{K}$. 
Through the intervening principle of "Strong inhibition of the same time, support the weak" (抑强扶弱), decrease the energy of the mother $s_{X}$ of $x$ while increase the energy of the bane $X_{K}$ of $s_{X}$.

\section{ACKNOWLEDGEMENTS}

This article has been repeatedly invited as reports, such as People's University of China in medical meetings, Shanxi University, Liaocheng University, Xuchang College, and so on. The work was supported by Specialized Research Fund for the Doctoral Program of Higher Education of Ministry of Education of China (Grant No. 200802691021).

Proof of Theorem 2.1. Let

\section{APPENDIX}

$\min =-0.1, \max =0.65, t_{0}=0.031359$.

(1) Firstly, from Eqs.(3) and $\rho(x)=\frac{1 / 2}{\lambda(x)+1 / 2} \Leftrightarrow \frac{\rho(x)}{1-\rho(x)}=\frac{1 / 2}{\lambda(x)} \Leftrightarrow \lambda(x)=\frac{1-\rho(x)}{2 \rho(x)}$.

(4), by equal ratio theorem,

If $x \rightarrow \min$ or $x \rightarrow \max$, then $\lambda(x) \rightarrow+\infty$, and $\rho(x) \rightarrow 0$. If $x=t_{0}$, then $\lambda(x)=0$, and $\rho(x)=1$. Thus $0<\rho(x) \leq 1$ and $0 \leq \lambda(x)<+\infty$ will be equivalent to each other. It is because $\lambda(x)$ is a monotone decreasing function of $x$ if $x \in\left(\mathrm{min}, t_{0}\right]$ or a monotone increasing function of $x$ if $x \in\left[t_{0}, \max \right)$; and $\rho(x)$ is a monotone decreasing function of $\lambda(x)$ if $\lambda(x) \in[0,+\infty)$; and $\lambda(x)$ is a monotone decreasing function of $\rho(x)$ if $\rho(x) \in(0,1]$.

In fact, $\lambda(x)$ is a monotone decreasing function of $x$ if $x \in\left(\mathrm{min}, t_{0}\right]$ since

$$
\begin{aligned}
\frac{d \lambda(x)}{d x} & =\frac{-(x-\min )(\max -x)-\left(t_{0}-x\right)[(\max -x)-(x-\min )]}{[(x-\min )(\max -x)]^{2}} \\
& =-\frac{(x-\min )(\max -x)+\left(t_{0}-x\right)[(\max -x)-(x-\min )]}{[(x-\min )(\max -x)]^{2}} \\
& =-\frac{\left[(x-\min )\left(\max -t_{0}\right)+\left(t_{0}-x\right)(\max -x)\right]}{[(x-\min )(\max -x)]^{2}}<0 .
\end{aligned}
$$

And $\lambda(x)$ is a monotone increasing function of $x$ if $x \in\left[t_{0}\right.$, max) since $\frac{d \lambda(x)}{d x}=\frac{(x-\min )(\max -x)-\left(x-t_{0}\right)[(\max -x)-(x-\min )]}{[(x-\min )(\max -x)]^{2}}$

$$
\begin{aligned}
& =\frac{(x-\min )(\max -x)-\left(x-t_{0}\right)[(\max -x)-(x-\min )]}{[(x-\min )(\max -x)]^{2}} \\
& =\frac{\left[(\max -x)\left(t_{0}-\min \right)+\left(x-t_{0}\right)(x-\min )\right]}{[(x-\min )(\max -x)]^{2}}>0 .
\end{aligned}
$$

And $\rho(x)$ is a monotone decreasing function of $\lambda(x)$ if $\lambda(x) \in[0,+\infty)$ since $\frac{d \rho(x)}{d \lambda(x)}=\frac{-(1 / 2)[1]}{[\lambda(x)+1 / 2]^{2}}=\frac{-1}{2[\lambda(x)+1 / 2]^{2}}<0$.

And $\lambda(x)$ is a monotone decreasing function of $\rho(x)$ if $\rho(x) \in(0,1]$ since $\frac{d \lambda(x)}{d \rho(x)}=\frac{1}{2}\left(\frac{(-1) \rho(x)-(1-\rho(x))[1]}{\rho(x)^{2}}\right)=\frac{-1}{2 \rho(x)^{2}}<0$. 
(2). Let the function $f(u)=\left(u+u^{3}\right)-\left(1-u^{3}\right), u \in(0,1]$. Then $f(u)$ is a monotone increasing function of $u$ since $f(u)^{\prime}=1+6 u^{2}>0$.

On the other hand, from [20], there is $f\left(\rho_{0}\right)=\left(\rho_{0}+\rho_{0}^{3}\right)-\left(1-\rho_{0}^{3}\right)=0$. Hence, if $\rho(x) \geq \rho_{0}$, then $f(\rho(x))=\left(\rho(x)+\rho(x)^{3}\right)-\left(1-\rho(x)^{3}\right) \geq f\left(\rho_{0}\right)=0$ because of monotonicity of $f$. Thus $\frac{1-\rho(x)}{2 \rho(x)} \leq \rho(x)^{2}$.

By the result of (1), there is $\lambda(x)=\frac{1-\rho(x)}{2 \rho(x)} \leq \frac{1-\rho_{0}}{2 \rho_{0}}=\rho_{0}^{2} \leq \rho(x)^{2}$ since $\lambda(x)$ is a monotone decreasing function of $\rho(x)$ where $0<\rho_{0} \leq \rho(x)$.

Similarly, there is

$\frac{\lambda(x)}{\rho(x)}=\frac{1-\rho(x)}{2 \rho(x)^{2}} \leq \frac{1-\rho_{0}}{2 \rho_{0}^{2}}=\rho_{0} \leq \rho(x)$

since $h(\rho(x))=\frac{\lambda(x)}{\rho(x)}$ is a monotone decreasing function of $\rho(x)$ where $0<\rho_{0} \leq \rho(x)$.

The proof of the monotone decreasing function $h(\rho(x))=\frac{\lambda(x)}{\rho(x)}$ is from

$\frac{d h(\rho(x))}{d \rho(x)}=\frac{1}{2}\left(\frac{(-1) \rho(x)^{2}-(1-\rho(x))[2 \rho(x)]}{\rho(x)^{4}}\right)=\frac{\rho(x)-2}{2 \rho(x)^{3}}<0$.

Furthermore, there is

$$
\frac{\lambda(x)}{\rho(x)^{2}}=\frac{1-\rho(x)}{2 \rho(x)^{3}} \leq \frac{1-\rho_{0}}{2 \rho_{0}^{3}}=1
$$

since $h(\rho(x))=\frac{\lambda(x)}{\rho(x)^{2}}$ is a monotone decreasing function of $\rho(x)$ where $0<\rho_{0} \leq \rho(x)$. The proof of the monotone decreasing function $h(\rho(x))=\frac{\lambda(x)}{\rho(x)^{2}}$ is from $\frac{d h(\rho(x))}{d \rho(x)}=\frac{1}{2}\left(\frac{(-1) \rho(x)^{3}-(1-\rho(x))\left[3 \rho(x)^{2}\right]}{\rho(x)^{6}}\right)=\frac{2 \rho(x)-3}{2 \rho(x)^{4}}<0$.

(3). It is similar to the proof of (2).

(4). Let the following function $g(u)=\left(u+c u^{3}\right)-\left(1-c u^{3}\right), \forall u, c \in(0,1]$.

Then $g(u)$ is a monotone increasing function of $u$ since $g(u)^{\prime}=1+6 c u^{2}>0$ where $0<c \leq 1$. On the other hand, $g(u)=2(c-1) u^{3}+f(u)$, where $f(u)=\left(u+u^{3}\right)-\left(1-u^{3}\right), u \in(0,1]$ is the same as that in (2). Therefore, if $\rho(x)<\rho_{0}$, then $g(\rho(x))<0,|g(\rho(x))|>2(1-c) \rho_{0}^{3}$, since

$g(\rho(x))<g\left(\rho_{0}\right)=2(c-1) \rho_{0}^{3}+f\left(\rho_{0}\right)=2(c-1) \rho_{0}^{3} \leq 0$.

The condition $g(\rho(x))<0$ implies that $\left(\rho_{1}+\rho_{2} \rho_{3}\right)=\rho(x)+c \rho(x)^{3}<1-c \rho(x)^{3}=1-\rho_{2} \rho_{3}$. 
(5). Consider the function $g(u)=2 c u^{3}-(1-u)$ is the same as in (4). Easy to know that $g(u)=0$ if and only if $c=\frac{1-u}{2 u^{3}}$. Denoted the function $\frac{1-u}{2 u^{3}}$ by $c(u)$. The $c(u)=\frac{1-u}{2 u^{3}}$ is a monotone decreasing function of $u \in(0,1]$ since

$c(u)^{\prime}=\frac{1}{2}\left(-3 u^{-4}+2 u^{-3}\right)=\frac{1}{2 u^{4}}(-3+2 u)<0, \forall, u \in(0,1]$.

If $c \geq c(u)$, i.e., $c \geq \frac{1-u}{2 u^{3}}$, then $g(u)=2 u^{3}\left(c-\frac{1-u}{2 u^{3}}\right) \geq 0$ which implies $\left(u+c u^{3}\right) \geq\left(1-c u^{3}\right)$.

If $0 \leq c<c(u)$, i.e., $0 \leq c<\frac{1-u}{2 u^{3}}$, then $g(u)=2 u^{3}\left(c-\frac{1-u}{2 u^{3}}\right)<0$ which implies $\left(u+c u^{3}\right)<\left(1-c u^{3}\right)$.

Because the balance solution of $\frac{1-u}{2 u^{3}}=\frac{1}{2}$ is $u=\rho_{0}^{\prime}$ and $c(u)=\frac{1-u}{2 u^{3}}$ is a monotone decreasing function of $u \in\left(\rho_{0}, 1\right]$, there are,

$u \in\left[\rho_{0}, \rho_{0}^{\prime}\right) \Leftrightarrow 1 \geq \frac{1-u}{2 u^{3}}>\frac{1}{2}$; and $u \in\left[\rho_{0}^{\prime}, 1\right) \Leftrightarrow 0<\frac{1-u}{2 u^{3}} \leq \frac{1}{2}$.

Thus one is that $|g(u)|=\left|2 c u^{3}+u-1\right| \leq\left(\rho_{0}^{\prime}\right)^{3}=0.31767, \forall u \in\left[\rho_{0}^{\prime}, 1\right)$ if $0 \leq c<\frac{1-u}{2 u^{3}} \leq \frac{1}{2}$ since $|g(u)|=2 u^{3}\left(\frac{1-u}{2 u^{3}}-c\right)<2 u^{3}\left(\frac{1-u}{2 u^{3}}\right)=1-u \leq 1-\rho_{0}^{\prime}=\left(\rho_{0}^{\prime}\right)^{3}=0.31767$.

And two is that

$|g(u)|=\left|2 c u^{3}+u-1\right| \leq 2 \rho_{0}^{3}=0.41024, \forall u \in\left[\rho_{0}, \rho_{0}^{\prime}\right)$ if

$0 \leq c \leq \frac{1}{2}<\frac{1-u}{2 u^{3}} \leq 1$ since

$|g(u)|=2 u^{3}\left(\frac{1-u}{2 u^{3}}-c\right)<2 u^{3}\left(\frac{1-u}{2 u^{3}}\right)=1-u \leq 1-\rho_{0}=2 \rho_{0}^{3}=0.41024$.

And three is that

$|g(u)|=\left|2 c u^{3}+u-1\right| \leq 0.31767, \forall u \in\left[\rho_{0}, \rho_{0}^{\prime}\right)$ if $\frac{1}{2}<c<\frac{1-u}{2 u^{3}} \leq 1$ since

$|g(u)|=\left|2 u^{3}\left(c-\frac{1-u}{2 u^{3}}\right)\right| \leq 2 u^{3} \cdot \frac{1}{2}=u^{3} \leq\left(\rho_{0}^{\prime}\right)^{3}=0.31767$.

In particular, from the proof of (3), there is that $g(u)=2 u^{3}\left(c-\frac{1-u}{2 u^{3}}\right)$ is a monotone increasing function of $u \in(0,1]$. When $c$ is nearly to $1 / 2$, not only the following results can be obtained directly $\rho_{1}-\rho_{3}=\rho(x)(1-c) \rightarrow \rho(x) / 2, \rho_{2}-\rho_{1} \rho_{3}=\rho(x)^{2}(1-c) \rightarrow \rho(x)^{2} / 2$, but also the following statements can be obtained.

(a). $g\left(\rho_{0}^{\prime}\right)=-(1-2 c)\left(\rho_{0}^{\prime}\right)^{3}$ and $g(u)=2 u^{3}\left(c-\frac{1-u}{2 u^{3}}\right)$ are nearly to 0 if $0<c<\frac{1-u}{2 u^{3}} \leq \frac{1}{2}$ in which $u \in\left[\rho_{0}^{\prime}, 1\right)$.

(b). $g\left(\rho_{0}\right)=-2(1-c) \rho_{0}^{3}$ and $g\left(\rho_{0}^{\prime}\right)=-(1-2 c)\left(\rho_{0}^{\prime}\right)^{3}$ are nearly to $-\rho_{0}^{3}=-0.20512,0$ respectively, if $0<c \leq \frac{1}{2}<\frac{1-u}{2 u^{3}} \leq 1$ in which $u \in\left[\rho_{0}, \rho_{0}^{\prime}\right)$. 
(c). $g\left(\rho_{0}\right)=-2(1-c) \rho_{0}^{3}$ and $\quad g\left(\rho_{0}^{\prime}\right)=-(1-2 c)\left(\rho_{0}^{\prime}\right)^{3}$ are nearly to $-\rho_{0}^{3}=-0.20512,0$ respectively, if $\frac{1}{2}<c<\frac{1-u}{2 u^{3}} \leq 1$ in which $u \in\left[\rho_{0}, \rho_{0}^{\prime}\right)$.

Taking $u=\rho(x)$, the desired are gotten.\#

Proof of Corollary 2.1.Let

$\min =-0.1, \max =0.65, t_{0}=0.031359$.

(1). From the proof of (1) in Theorem 2.1, for any $0<d<1$, there is an unique solution $x \in\left(\min , t_{0}\right)$ and there is also an unique solution $v \in\left(t_{0}, \max \right)$, such that

$$
\lambda\left(t_{0}\right)=0 \leq \lambda(u)=\lambda(v)=(1-d) /(2 d),
$$

since $\lambda(x)$ is a monotone decreasing function of $x \in\left(\min , t_{0}\right)$ or a monotone increasing function of $x \in\left(t_{0}, \max \right)$ if $0 \leq(1-d) /(2 d)<+\infty$.

By the result of (1) in Theorem 2.1, $\lambda(u)=\lambda(v)=(1-d) /(2 d)$ is equivalent to $\rho(u)=\frac{1 / 2}{\lambda(u)+1 / 2}=d=\frac{1 / 2}{\lambda(v)+1 / 2}=\rho(v)$.

For $d=\rho_{0}, \lambda(u)=\lambda(v)=\left(1-\rho_{0}\right) /\left(2 \rho_{0}\right)=\rho_{0}^{2}$ since $\left(\rho_{0}+\rho_{0}^{3}\right)=\left(1-\rho_{0}^{3}\right)$.

For $d=\rho_{0}^{\prime}, \lambda(u)=\lambda(v)=\left(1-\rho_{0}^{\prime}\right) /\left(2 \rho_{0}^{\prime}\right)=\left(\rho_{0}^{\prime}\right)^{2} / 2$, since $\left(\rho_{0}^{\prime}\right)^{3}=1-\rho_{0}^{\prime}$.

The others can be obtained through the calculated directly from the result of (1) by SAS. \#

Proof of Theorem 3.2 Let

$$
a=7.7473 \%, b=18.460 \%, t_{0}=12.269 \% .
$$

(1).If a subsystem $x$ of a steady multilateral system $v$ falls a virtual disease, denoted by $\downarrow^{0}$, which means that there is a force such that its energy $\varphi(X)$ decreases, i.e., changed by the increment $\Delta \varphi(X)=-\Delta<0$. By the Intervention Rule in [20], its capability of intervention reaction can make the mother $s_{x}$ of $x$ to fall a virtual disease by a negative increment $\Delta \varphi\left(S_{X}\right)=-\rho_{1} \Delta<0$, in which its absolute is large, denoted by $\downarrow^{+}$, and also can make the son $X_{S}$ of $X$ to fall a virtual disease by a negative increment $\Delta \varphi\left(X_{S}\right)=-\rho_{2} \Delta<0$, in which its absolute is small, denoted by $\downarrow$ - Diseases of the loving subsystems have the same direction of $x$.

At the same time, its capability of intervention reaction can make the bane $K_{x}$ of $x$ to fall a real disease by a large increment $\Delta \varphi\left(K_{X}\right)=\rho_{1} \Delta>0$, denoted by $\uparrow^{+}$, and also can make the prisoner $X_{K}$ of $x$ to fall a real disease by a little increment $\Delta \varphi\left(X_{K}\right)=\rho_{2} \Delta>0$, denoted by $\uparrow^{\uparrow}$. Diseases of the killing subsystems are the opposite direction of $x$.

The bane $K_{X}$ of $x$ is the biggest victim since its increment $\Delta \varphi\left(K_{X}\right)=\rho_{1} \Delta>0$ is large.

On the other hand, if the PPI inflation rate $x \in[a, b]$, by Corollary 2.1 , there is $1 \geq \rho_{1}=\rho(x) \geq \rho_{0}$ such that

$$
\rho_{1}+\rho_{2} \rho_{3}=\rho(x)+c \rho(x)^{3} \geq 1-\rho_{2} \rho_{3}=1-c \rho(x)^{3} .
$$

Thus, $c \geq[1-\rho(x)] /\left(2 \rho(x)^{3}\right)>0, \forall x \neq t_{0}$. It means that the economy is healthy such that there is the capability of self-protection of the steady multilateral system. By the Self-protection Rule in 
[20], its capability of self-protection can make the biggest victim as the bane $K_{x}$ of $x$ restored by a fixed increment $\Delta \varphi\left(K_{X}\right)_{1}=-\rho_{3} \Delta<0$, denoted by $\Downarrow$.

By the Intervention Rule in [20] again and from Theorem 3.1, its capability of self-protection can make the mother $X_{K}$ of $K_{X}$ to fall a virtual disease by a negative increment $\Delta \varphi\left(X_{K}\right)_{1}=-\rho_{1} \rho_{3} \Delta<0$, in which its absolute is large, denoted by $\Downarrow^{+}$, and also can make the son $S_{X}$ of $K_{X}$ to fall a virtual disease by a negative increment $\Delta \varphi\left(S_{X}\right)_{1}=-\rho_{2} \rho_{3} \Delta<0$, in which its absolute is small, denoted by $\Downarrow^{-}$. Diseases of the loving subsystems have the same direction of $K_{x}$.

At the same time, its capability of self-protection can make the bane $X_{S}$ of $K_{X}$ to fall a real disease by a large increment $\Delta \varphi\left(X_{S}\right)_{1}=\rho_{1} \rho_{3} \Delta>0$, denoted by $\Uparrow^{+}$, and also can make the prisoner $x$ of $K_{x}$ to fall a real disease by a little increment $\Delta \varphi(X)_{1}=\rho_{2} \rho_{3} \Delta>0$, denoted by $\Uparrow^{-}$. Diseases of the killing subsystems are the opposite direction of $K_{x}$. All these changes consist of the following Figure 2 .

From Figure 2 or from Theorem 3.2 in [20], if $\rho_{3}=\rho_{1}=\rho(x)$, i.e., $c=1$, its capabilities of intervention reaction and self-protection can make the subsystems $X_{S}, X_{K}$ and $K_{X}$ be restored at the same time, but the subsystems $x$ and $s_{x}$ will decrease their energies. In mathematics, from Theorems 3.1 and 3.2 in [20] , the final increments can be obtained as follows:

$\Delta \varphi(X)_{2}=\Delta \varphi(X)_{1}+\Delta \varphi(X)=-\left(1-\rho_{2} \rho_{3}\right) \Delta=-\left(1-c \rho(x)^{3}\right) \rightarrow-\left(1-\rho(x)^{3}\right)<0$,

$\Delta \varphi\left(S_{X}\right)_{2}=\Delta \varphi\left(S_{X}\right)_{1}+\Delta \varphi\left(S_{X}\right)=-\left(\rho_{1}+\rho_{1} \rho_{3}\right) \Delta=-\left(\rho(x)+c \rho(x)^{2}\right) \Delta$

$\rightarrow-\left(\rho(x)+\rho(x)^{2}\right) \Delta<0$,

$\Delta \varphi\left(K_{X}\right)_{2}=\Delta \varphi\left(K_{X}\right)_{1}+\Delta \varphi\left(K_{X}\right)=\left(\rho_{1}-\rho_{3}\right) \Delta=\rho(x)(1-c) \Delta \rightarrow 0+$,

$\Delta \varphi\left(X_{K}\right)_{2}=\Delta \varphi\left(X_{K}\right)_{1}+\Delta \varphi\left(X_{K}\right)=\left(\rho_{2}-\rho_{1} \rho_{3}\right) \Delta=\rho(x)^{2}(1-c) \Delta \rightarrow 0+$,

$\Delta \varphi\left(X_{S}\right)_{2}=\Delta \varphi\left(X_{S}\right)_{1}+\Delta \varphi\left(X_{S}\right)=-\left(\rho_{2}-\rho_{1} \rho_{3}\right) \Delta=-\rho(x)^{2}(1-c) \Delta \rightarrow 0-$.

It means that the first physiological system of the steady multilateral system runs normally. From the idea of TCE, by the Affording Rule, the second physiological system of the steady multilateral system will not work to afford for. It is because there is a full Yin food $\Delta \varphi(X)_{2}=-\left(1-\rho_{2} \rho_{3}\right) \Delta=\left(1-c \rho(x)^{3}\right) \Delta<0$ for the second physiological system if the subsystem $X$ falls a virtual disease or is intervened for a healthy economy by the Attaining Rule.

By Theorem 2.1 and Corollary 2.1, the PPI inflation rate $x \in[a, b]$ makes $_{1 \geq \rho(x) \geq \rho_{0}}$ such that $-\left(\rho(x)+\rho(x)^{3}\right) \leq-\left(1-\rho(x)^{3}\right)$, therefore the first transfer law is the first occurrence of the virtual disease of the mother $s_{x}$ of $x$ if a subsystem $x$ of a steady multilateral system $V$ falls a virtual disease.

From Figure 2 and by Definition 3.1, the relation disease between virtual $s_{x}$ and virtual $x$ is less serious since virtual $s_{x}$ can love virtual $x$.

If the disease continues to develop, it will lead to undermine firstly the best state of the capability of self-protection of the steady multilateral system, i.e., $c \neq 1$, since any disease that causes side effects in the first place dues to the non-best state of self-protection ability, i.e., $\rho_{3}=c \rho(x)<\rho(x)=\rho_{1}$, where $0 \leq c<1$, even if the treatment principle of TCE is used. Thus the self-protection coefficient $\rho_{3}$ will near to small such that firstly $\left(\rho_{1}-\rho_{3}\right)=\rho(x)(1-c)>0$, secondly $\left(\rho_{2}-\rho_{1} \rho_{3}\right)=\rho(x)^{2}(1-c)>0$, and finally $-\left(\rho_{2}-\rho_{1} \rho_{3}\right)=-\rho(x)^{2}(1-c)<0$. 
By Theorem 3.1 in [20], the victim $K_{x}$ or $X_{K}$ of $x$ will encounter a sick which is different from the disease direction of $x$, i.e., changed by the increments

$\Delta \varphi\left(K_{X}\right)_{2}=\Delta \varphi\left(K_{X}\right)+\Delta \varphi\left(K_{X}\right)_{1}=\left(\rho_{1}-\rho_{3}\right) \Delta=\rho(x)(1-c) \Delta>0, \Delta \varphi\left(X_{K}\right)_{2}=\Delta \varphi\left(X_{K}\right)+\Delta \varphi\left(X_{K}\right)_{1}=\left(\rho_{2}-\rho_{1} \rho_{3}\right) \Delta=\rho(x)^{2}(1-c) \Delta>0$,

respectively. At the same time, the son $X_{S}$ of $x$ also falls a virtual ill which is the same disease direction of $x$, i.e., changed by the increment

$\Delta \varphi\left(X_{S}\right)_{2}=\Delta \varphi\left(X_{S}\right)+\Delta \varphi\left(X_{S}\right)_{1}=-\left(\rho_{2}-\rho_{1} \rho_{3}\right) \Delta=-\rho(x)^{2}(1-c) \Delta<0$,

since $1>c \geq 0$. Therefore the transfer law is secondly the real disease of the bane $K_{x}$ of $x$ after the virtual disease of the mother $s_{X}$ of $X$, and thirdly the real disease of the prisoner $X_{K}$ of $X$, and fourthly the virtual disease of the son $X_{S}$ of $x$, against the loving relationship order transmission.

In fact, watching Figure 2, $K_{x}$ is real, $X_{K}$ is real, $X_{S}$ is virtual, $x$ is virtual, and $s_{X}$ is virtual. The order of the transfer law of diseases must be against the loving relationship order transmission. It is because

$\left(\rho_{1}-\rho_{3}\right) \geq\left(\rho_{2}-\rho_{1} \rho_{3}\right) \geq-\left(\rho_{2}-\rho_{1} \rho_{3}\right) \geq-\left(\rho_{1}-\rho_{2} \rho_{3}\right) \geq-\left(\rho_{1}+\rho_{2} \rho_{3}\right)$.

The result is obtained from $\left(1-\rho_{2} \rho_{3}\right) \leq\left(\rho_{1}+\rho_{2} \rho_{3}\right)$ since

$\left(\rho_{1}+\rho_{2} \rho_{3}\right) \geq\left(\rho_{1}-\rho_{2} \rho_{3}\right) \geq\left(\rho_{1}-\rho_{3}\right)=\rho(x)(1-c)$

$\geq \rho(x)^{2}(1-c)=\left(\rho_{2}-\rho_{1} \rho_{3}\right)$.

In other words, the diseases are transferred from real $K_{x}$ to virtual $s_{x}$ against the loving relationship order if $x$ is the cause of diseases or $x$ is intervened. It implies that the mother $s_{x}$ of $x$ will obtain the best Yin (negative) energy if the energy of $x$ decreases.

Watching Figure 2 and by Definitions 3.1 and 3.2, the relation disease between real $K_{x}$ and virtual $x$ is less serious since real $K_{x}$ can kill virtual $x$, but the relation disease between virtual $x$ and real $X_{K}$ is more serious since virtual $X$ cannot kill real $X_{K}$ which will destroy the killing order from $X$ to $X_{K}$ of the Yin Yang Wu Xing system if the disease continues to develop.

Finally the relation disease between virtual $X_{S}$ and virtual $x$ is also less serious since virtual $x$ can love virtual $X_{S}$. The less serious relation disease between virtual $X_{S}$ and virtual $x$ makes that the loving order from $x$ to $X_{S}$ will be restored easily. The transferring way of strengthening the loving relationship can make the new virtual disease of $x$ remission since $\Delta \varphi(X)_{2}=-\left(1-c \rho(x)^{3}\right) \Delta \geq-\Delta=\Delta \varphi(X)$.

Despite of the positive way, the system will be still for the next round of disease transmission, until disease rehabilitation.

(2). If a subsystem $x$ of a steady multilateral system $V$ falls a real disease, denoted by $\uparrow^{0}$, which means that there is a force such that its energy $\varphi(X)$ increases, i.e., changed by the increment $\Delta \varphi(X)=\Delta>0$. By the Intervention Rule in [20], its capability of intervention reaction can make the son $X_{S}$ of $x$ to fall a real disease by a large increment $\Delta \varphi\left(X_{S}\right)=\rho_{1} \Delta>0$, denoted by $\uparrow^{+}$, and also can make the mother $S_{X}$ of $X$ to fall a real disease by a little 
increment $\Delta \varphi\left(S_{X}\right)=\rho_{2} \Delta>0$, denoted by $\uparrow^{-}$. Diseases of the loving subsystems have the same direction of $x$.

At the same time, its capability of intervention reaction can make the prisoner $X_{k}$ of $x$ to fall a virtual disease by a negative increment $\Delta \varphi\left(X_{K}\right)=-\rho_{1} \Delta<0$, in which its absolute is large, denoted by $\downarrow^{+}$, and also can make the bane $K_{x}$ of $x$ to fall a virtual disease by a negative increment $\Delta \varphi\left(K_{X}\right)=-\rho_{2} \Delta<0$, in which its absolute is small, denoted by $\downarrow^{-}$. Diseases of the killing subsystems are the opposite direction of $x$.

The prisoner $X_{K}$ of $x$ is the biggest victim since its increment $\Delta \varphi\left(X_{K}\right)=-\rho_{1} \Delta<0$ is the scope of big changes.

On the other hand, if the PPI inflation rate $x \in[a, b]$, by Theorem 2.1 and Corollary 2.1, there is $1 \geq \rho_{1}=\rho(x) \geq \rho_{0}$ such that $\rho_{1}+\rho_{2} \rho_{3}=\rho(x)+c \rho(x)^{3} \geq 1-\rho_{2} \rho_{3}=1-c \rho(x)^{3}$.

Thus, $c \geq[1-\rho(x)] /\left(2 \rho(x)^{3}\right)>0, \forall x \neq t_{0}$. It means that the economy is healthy such that there is a good capability of self-protection of the steady multilateral system.

By the Self-protection Rule in [20], its capability of self-protection can make the biggest victim as the prisoner $X_{K}$ of $x$ restored by a fixed increment $\Delta \varphi\left(X_{K}\right)_{1}=\rho_{3} \Delta>0$, denoted by $\Uparrow^{0}$.

By the Intervention Rule in [20] again and from Theorem 3.1, its capability of self-protection can make the son $K_{X}$ of $X_{K}$ to fall a real disease by a large increment $\Delta \varphi\left(K_{X}\right)_{1}=\rho_{1} \rho_{3} \Delta>0$, denoted by $\Uparrow^{+}$, and also can make the mother $X_{S}$ of $X_{K}$ to fall a real disease by a little increment $\Delta \varphi\left(X_{S}\right)_{1}=\rho_{2} \rho_{3} \Delta>0$, denoted by $\Uparrow^{-}$. Diseases of the loving subsystems have the same direction of $X_{K}$.

At the same time, its capability of self-protection can make the prisoner $s_{X}$ of $X_{K}$ to fall a virtual disease by a negative increment $\Delta \varphi\left(S_{X}\right)_{1}=-\rho_{1} \rho_{3} \Delta<0$, in which its absolute is large, denoted by $\Downarrow^{+}$, and also can make the bane $x$ of $X_{K}$ to fall a virtual disease by a negative increment $\Delta \varphi(X)_{1}=-\rho_{2} \rho_{3} \Delta<0$, in which its absolute is small, denoted by $\Downarrow^{-}$. Diseases of the killing subsystems are the opposite direction of $X_{\mathrm{R}^{*}}$. All these changes consist of the following Figure 3. From Figure 3 or by Theorem 3.2 in [20], if $\rho_{3}=\rho_{1}=\rho(x)$, i.e., $c \rightarrow 1$, its capabilities of intervention reaction and self-protection can make the subsystems $s_{x}, K_{x}$ and $X_{K}$ restored at the same time, but the subsystems $x$ and $X_{S}$ will increase their energies. In mathematics, from Theorems 3.1 and 3.2 in [20] , the final increments can be obtained as follows:

$\Delta \varphi(X)_{2}=\Delta \varphi(X)_{1}+\Delta \varphi(X)=\left(1-\rho_{2} \rho_{3}\right) \Delta=\left(1-c \rho(x)^{3}\right) \Delta \rightarrow\left(1-\rho(x)^{3}\right) \Delta>0$,

$\Delta \varphi\left(X_{S}\right)_{2}=\Delta \varphi\left(X_{S}\right)_{1}+\Delta \varphi\left(X_{S}\right)=\left(\rho_{1}+\rho_{1} \rho_{3}\right) \Delta=\left(\rho(x)+c \rho(x)^{2}\right) \Delta \rightarrow\left(\rho(x)+\rho(x)^{2}\right) \Delta>0$,

$\Delta \varphi\left(X_{K}\right)_{2}=\Delta \varphi\left(X_{K}\right)_{1}+\Delta \varphi\left(X_{K}\right)=-\left(\rho_{1}-\rho_{3}\right) \Delta=-\rho(x)(1-c) \Delta \rightarrow 0-$,

$\Delta \varphi\left(K_{X}\right)_{2}=\Delta \varphi\left(K_{X}\right)_{1}+\Delta \varphi\left(K_{X}\right)=-\left(\rho_{2}-\rho_{1} \rho_{3}\right) \Delta=-\rho(x)^{2}(1-c) \Delta \rightarrow 0-$,

$\Delta \varphi\left(S_{X}\right)_{2}=\Delta \varphi\left(S_{X}\right)_{1}+\Delta \varphi\left(S_{X}\right)=\left(\rho_{2}-\rho_{1} \rho_{3}\right) \Delta=\rho(x)^{2}(1-c) \Delta \rightarrow 0+$

It means that the first physiological system of the steady multilateral system runs normally. From the idea of TCE, by the Affording Rule, the second physiological system of the steady multilateral system will not work to afford for. It is because there is a full Yang food 
$\Delta \varphi(X)_{2}=\left(1-\rho_{2} \rho_{3}\right) \Delta=\left(1-c \rho(x)^{3}\right) \Delta>0$ for the second physiological system if the subsystem $X$ falls a real disease or is intervened for a healthy economy by the Attaining Rule.

By Theorem 2.1 and Corollary 2.1, the PPI inflation rate $x \in[a, b]$ makes $1 \geq \rho_{1}=\rho(x) \geq \rho_{0}$ such that $\left(\rho(x)+\rho(x)^{3}\right) \geq\left(1-\rho(x)^{3}\right)$. Therefore the transfer law is the first occurrence of the real disease of the son $X_{S}$ of $x$ if a subsystem $x$ of a steady multilateral system $v$ falls a real disease. From Figure 3 and by Definition 3.1, the relation disease between real $x$ and real $X_{S}$ is less serious since real $x$ can love real $X_{S}$.

If the disease continues to develop, it will lead to undermine firstly the capability of selfprotection of the steady multilateral system, i.e., $c \neq 1$ since any disease that causes side effects in the first place dues to the non-best state of self-protection ability, i.e., $\rho_{3}=c \rho(x)<\rho(x)=\rho_{1}$ where $0 \leq c<1$, even if the treatment principle of TCE is used. Thus the self-protection coefficient $\rho_{3}$ will near to small such that firstly $\left(\rho_{1}-\rho_{3}\right)=\rho(x)(1-c)>0$, and secondly $\left(\rho_{2}-\rho_{1} \rho_{3}\right)=\rho(x)^{2}(1-c)>0$, and finally $-\left(\rho_{2}-\rho_{1} \rho_{3}\right)=-\rho(x)^{2}(1-c)<0$. By Theorem 3.1 in [20], the victim $X_{K}$ or $K_{X}$ of $X$ will encounter a sick respectively which is different from the disease direction of $X$, i.e., changed by the increments

$\Delta \varphi\left(X_{K}\right)_{2}=\Delta \varphi\left(X_{K}\right)+\Delta \varphi\left(X_{K}\right)_{1}=-\left(\rho_{1}-\rho_{3}\right) \Delta=-\rho(x)(1-c)<0$,

$\Delta \varphi\left(K_{X}\right)_{2}=\Delta \varphi\left(K_{X}\right)+\Delta \varphi\left(K_{X}\right)_{1}=-\left(\rho_{2}-\rho_{1} \rho_{3}\right) \Delta=-\rho(x)^{2}(1-c)<0$,

Respectively since $1>c \geq 0$. At the same time, the mother $s_{x}$ of $x$ also falls a real ill which is the same disease direction of $x$, i.e., changed by the increment

$\Delta \varphi\left(S_{X}\right)_{2}=\Delta \varphi\left(S_{X}\right)+\Delta \varphi\left(S_{X}\right)_{1}=\left(\rho_{2}-\rho_{1} \rho_{3}\right)=\rho(x)^{2}(1-c) \Delta>0$

since $1>c \geq 0$. Therefore the first transfer law is secondly the virtual disease of the prisoner $X_{K}$ of $x$ after the real disease of the son $X_{S}$ of $x$, and thirdly the virtual disease of the bane $K_{X}$ of $x$, and fourthly the real disease of the mother $s_{x}$ of $x$, along the loving relationship order transmission.

In fact, watching Figure 3, $X_{K}$ is virtual, $K_{X}$ is virtual, $s_{X}$ is real, $x$ is real, and $X_{S}$ is real. The order of the transfer law of diseases must be along the loving relationship order transmission. It is because

$-\left(\rho_{1}-\rho_{3}\right) \leq-\left(\rho_{2}-\rho_{1} \rho_{3}\right) \leq\left(\rho_{2}-\rho_{1} \rho_{3}\right) \leq\left(\rho_{1}-\rho_{2} \rho_{3}\right) \leq\left(\rho_{1}+\rho_{2} \rho_{3}\right)$.

The result is obtained from $\left(1-\rho_{2} \rho_{3}\right) \leq\left(\rho_{1}+\rho_{2} \rho_{3}\right)$ since

$\left(\rho_{1}+\rho_{2} \rho_{3}\right) \geq\left(\rho_{1}-\rho_{2} \rho_{3}\right) \geq\left(\rho_{1}-\rho_{3}\right)=\rho(x)(1-c)$

$\geq \rho(x)^{2}(1-c)=\left(\rho_{2}-\rho_{1} \rho_{3}\right)$.

In other words, the diseases are transferred from virtual $X_{K}$ to real $X_{S}$ along the loving relationship order if $x$ is the cause of diseases or $x$ is intervened. It implies that the son $X_{S}$ of $x$ will obtain the best Yang (positive) energy if the energy of $x$ increases.

Watching Figure 3 and by Definitions 3.1 and 3.2, the relation disease between real $x$ and virtual $X_{K}$ is less serious since real $x$ can kill virtual $X_{K}$, but the relation disease between virtual $K_{X}$ and real $x$ is more serious since virtual $K_{X}$ cannot kill real $x$ which will destroy the killing order of the Yin Yang Wu Xing system from $K_{x}$ to $x$. 
Finally the relation disease between $s_{X}$ and $x$ is also less serious since real $s_{X}$ can love real $x$ The less serious relation disease between real $s_{x}$ and real $x$ makes that the loving order from $s_{x}$ to $x$ will be restored easily. The transferring way of strengthening the loving relationship can make the real disease of $x$ remission since $\Delta \varphi(X)_{2}=\left(1-c \rho(x)^{3}\right) \Delta<\Delta=\Delta \varphi(X)$. Despite of the positive way, the system will be still for the next round of disease transmission, until disease rehabilitation. This completes the proof.\#

Proof of Theorem 3.3. Let

$$
a=7.7473 \%, b=18.460 \%, t_{0}=12.269 \% \text {. }
$$

(1).If a subsystem $x$ of a steady multilateral system $v$ falls a virtual disease, denoted by $\downarrow^{0}$, which means that there is a force such that its energy $\varphi(X)$ decreases, i.e., changed by the increment $\Delta \varphi(X)=-\Delta<0$. By the Intervention Rule in [20], its capability of intervention reaction can make the mother $s_{X}$ of $x$ to fall a virtual disease by a negative increment $\Delta \varphi\left(S_{X}\right)=-\rho_{1} \Delta<0$, in which its absolute is large, denoted by $\downarrow^{+}$, and also can make the son $X_{S}$ of $X$ to fall a virtual disease by a negative increment $\Delta \varphi\left(X_{S}\right)=-\rho_{2} \Delta<0$, in which its absolute is small, denoted by $\downarrow^{-}$. Diseases of the loving subsystems have the same direction of $x$.

At the same time, its capability of intervention reaction can make the bane $K_{x}$ of $x$ to fall a real disease by a large increment $\Delta \varphi\left(K_{X}\right)=\rho_{1} \Delta>0$, denoted by $\uparrow^{+}$, and also can make the prisoner $X_{K}$ of $x$ to fall a real disease by a little increment $\Delta \varphi\left(X_{K}\right)=\rho_{2} \Delta>0$, denoted by $\uparrow$. Diseases of the killing subsystems are the opposite direction of $x$.

The bane $K_{X}$ of $X$ is the biggest victim since its increment $\Delta \varphi\left(K_{X}\right)=\rho_{1} \Delta>0$ is large.

By Corollary 2.1 and Theorem 2.1, the PPI inflation rate $x \in[a, b]$ makes that $\rho_{0}>\rho_{1}=\rho(x)>\rho_{2}=\rho(x)^{2}>0$ such that $\left(\rho_{1}+\rho_{2} \rho_{3}\right)<\left(1-\rho_{2} \rho_{3}\right)$.In this case, the steady multilateral system $V$ is abnormal or unhealthy. The unhealthy means the lack of self-protection ability. Thus the self-protection coefficient $\rho_{3}=c \rho(x)$ will near to small such that firstly $0<\left(\rho_{1}-\rho_{3}\right)=\rho(x)(1-c) \rightarrow \rho(x)=\rho_{1}$ and secondly $0<\left(\rho_{2}-\rho_{1} \rho_{3}\right)=\rho(x)^{2}(1-c) \rightarrow \rho(x)^{2}=\rho_{2}$, i.e., $c \rightarrow 0$, if the disease continues to develop.

Consider in the worst state: $\rho_{3}=c \rho(x) \rightarrow 0$, i.e., $c \rightarrow 0$, where $\rho_{0}>\rho_{1}=\rho(x)>\rho_{2}=\rho(x)^{2}>0$. Its capability of self-protection cannot make the biggest victim as the bane $K_{x}$ of $X$ restored. In mathematics, from Theorems 3.1 and 3.2 in [20], the final increments can be obtained as follows:

$\Delta \varphi(X)_{2}=\Delta \varphi(X)_{1}+\Delta \varphi(X)=-\left(1-\rho_{2} \rho_{3}\right) \Delta=-\left(1-c \rho(x)^{3}\right) \Delta \rightarrow-\Delta<0$,

$\Delta \varphi\left(S_{X}\right)_{2}=\Delta \varphi\left(S_{X}\right)_{1}+\Delta \varphi\left(S_{X}\right)=-\left(\rho_{1}+\rho_{1} \rho_{3}\right) \Delta=-\left(\rho(x)+c \rho(x)^{2}\right) \Delta \rightarrow-\rho_{1} \Delta<0$,

$\Delta \varphi\left(K_{X}\right)_{2}=\Delta \varphi\left(K_{X}\right)_{1}+\Delta \varphi\left(K_{X}\right)=\left(\rho_{1}-\rho_{3}\right) \Delta=\rho(x)(1-c) \Delta \rightarrow \rho(x) \Delta=\rho_{1} \Delta>0$,

$\Delta \varphi\left(X_{K}\right)_{2}=\Delta \varphi\left(X_{K}\right)_{1}+\Delta \varphi\left(X_{K}\right)=\left(\rho_{2}-\rho_{1} \rho_{3}\right) \Delta=\rho(x)^{2}(1-c) \Delta \rightarrow \rho(x)^{2} \Delta=\rho_{2} \Delta>0$,

$\Delta \varphi\left(X_{S}\right)_{2}=\Delta \varphi\left(X_{S}\right)_{1}+\Delta \varphi\left(X_{S}\right)=-\left(\rho_{2}-\rho_{1} \rho_{3}\right) \Delta=-\rho(x)^{2}(1-c) \Delta \rightarrow-\rho_{2} \Delta<0$.

It means that the first physiological system of the steady multilateral system runs hardly, such that the final increments of self-protection are similar to the increments of intervention reaction. The hard running is not only because the killing order has been destroyed, but also the loving order cannot be running. 
From the idea of TCE, by the Affording Rule, the second physiological system of the steady multilateral system will work. It is because a steady multilateral system would become ill or dies if its Chi or energy in the steady multilateral system is imbalanced or exhausted. The purpose of the second physiological system working is to increase the energy of the system, and restores the order of the killing relationship of the Yin Yang Wu Xing system.

By Theorems 3.3 and 3.4 and Corollary 3.2 in [20], the method of "Strong inhibition of the same time, support the weak", i.e., to increase energy of virtual $x$ while to reduce energy of real $x_{K}$, should be used in this case: $x \bar{\in}[a, b]$ which is equivalent to that $\rho_{0}>\rho_{1}=\rho(x)>\rho_{2}=\rho(x)^{2}>0$, such that $\left(\rho_{1}+\rho_{2} \rho_{3}\right)<\left(1-\rho_{2} \rho_{3}\right)$.It is because the inequality $\left(\rho_{1}+\rho_{2} \rho_{3}\right)<\left(1-\rho_{2} \rho_{3}\right)$ makes the absolute value of their increments $\pm\left(1-\rho_{2} \rho_{3}\right) \Delta_{1}$ becoming large .

By Definitions 3.1 and 3.2, consider the lack of self-protection ability, from Figure 4, for a virtual $x$, its capability of intervention reaction can make many relation diseases occurrence. Only the relation between virtual $x$ and real $x_{K}$ can be considered as more serious, since virtual $x$ cannot kill real $x_{K}$, which will destroy the killing order from $x$ to $x_{K}$ of the Yin Yang Wu Xing system if the disease continues to develop.

By Affording Rule again, abiding by the treatment principle of "Strong inhibition of the same time, support the weak", the intervention of the second physiological system is to increase the energy of virtual $x$ while to reduce the energy of real $X_{K}$. If an intervention force on the subsystem $x$ of steady multilateral system $v$ is implemented such that its energy $\varphi(X)$ has been changed by an increment $\Delta \varphi(X)=\Delta_{1}>0$, denoted by $\Uparrow^{0}$, and at the same time, another intervention force on the subsystem $x_{K}$ of steady multilateral system $v$ is also implemented such that its energy $\varphi\left(X_{K}\right)$ has been changed by an increment $\Delta \varphi\left(X_{K}\right)=-\Delta_{1}<0$, denoted by $\Downarrow^{0}$, then from Theorem 3.4 in [20], all other subsystems: $s_{X}, K_{x}$ and $X_{S}$ can be restored at the same time, and the subsystems $x$ and $x_{K}$ will increase and decrease their energies by the same size but the direction opposite.

In mathematics, from Theorems 3.1, 3.3 and 3.4 in [20] , the final increments can be obtained as follows:

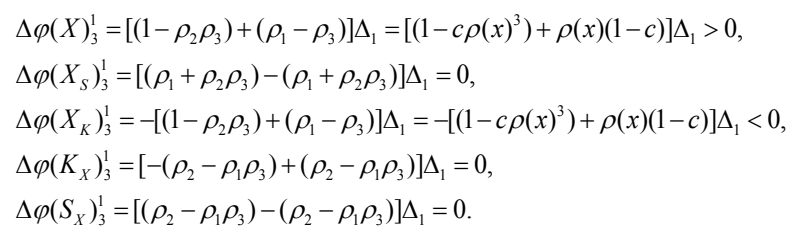

All these changes consist of the following Figure 4.

Of course, the virtual disease of $x$ comes from the son $X_{S}$ of $x$ since any virtual disease always comes from its son by Theorem 3.2 in [20].

In order to cure the virtual $x$, by Affording Rule, the increment $\Delta \varphi(X)_{3}^{1}=\left[\left(1-\rho_{2} \rho_{3}\right)+\left(\rho_{1}-\rho_{3}\right)\right] \Delta_{1}=\left(1-c \rho(x)^{3}+\rho(x)(1-c)\right) \Delta_{1}>0$ must be very big. For example $\Delta_{1} \geq \Delta$, which means righteousness to defeat evil, this treatment can make the increment $\Delta \varphi\left(X_{K}\right)_{3}^{1}=-\left[\left(1-\rho_{2} \rho_{3}\right)+\left(\rho_{1}-\rho_{3}\right)\right] \Delta_{1}=-\left(1-c \rho(x)^{3}+\rho(x)(1-c)\right) \Delta_{1}<0$ be very small. 
In fact, by Affording Rule and from Theorems 3.1-3.4 of [20], the all final increments can be obtained as follows:

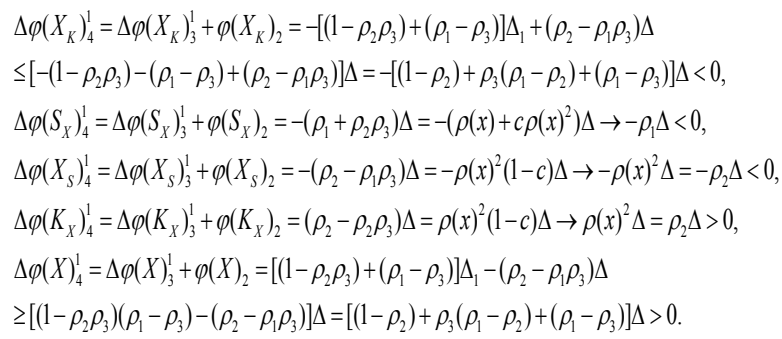

Therefore, the transfer law is the first occurrence of the virtual disease of the prisoner $X_{K}$ of $x$; secondly the virtual disease of the mother $s_{X}$ of $x$, thirdly the virtual disease of the son $X_{S}$ of $X$, and finally the real disease of the bane $K_{x}$ of $x$.

In fact, watching Figure $4, X_{K}$ is virtual, $s_{X}$ is virtual, $X_{S}$ is virtual, and $K_{X}$ is real. The order of the transfer law of diseases must be along the killing relationship order transmission. It is because $-\left[\left(1-\rho_{2}\right)+\rho_{3}\left(\rho_{1}-\rho_{2}\right)+\left(\rho_{1}-\rho_{3}\right)\right]<-\left(\rho_{1}+\rho_{2} \rho_{3}\right)<-\left(\rho_{2}-\rho_{1} \rho_{3}\right)<\left(\rho_{2}-\rho_{1} \rho_{3}\right)$ $<\left[\left(1-\rho_{2}\right)+\rho_{3}\left(\rho_{1}-\rho_{2}\right)+\left(\rho_{1}-\rho_{3}\right)\right]$.

The result is obtained from $\left(1-\rho_{2} \rho_{3}\right)>\left(\rho_{1}+\rho_{2} \rho_{3}\right)$ since

$\left[\left(1-\rho_{2}\right)+\rho_{3}\left(\rho_{1}-\rho_{2}\right)+\left(\rho_{1}-\rho_{3}\right)\right]-\left(\rho_{1}+\rho_{2} \rho_{3}\right)=1-\rho_{2} \rho_{3}+\rho_{3} \rho_{1}-\rho_{2} \rho_{3}-\rho_{2}$

$>\rho_{1}+\rho_{2} \rho_{3}+\rho_{3} \rho_{1}-\rho_{2} \rho_{3}-\rho_{2}=\left(\rho_{1}-\rho_{2}\right)+\rho_{3} \rho_{1}>0$,

and $\left(\rho_{1}+\rho_{2} \rho_{3}\right)-\left(\rho_{2}-\rho_{1} \rho_{3}\right)=\left(\rho_{1}-\rho_{2}\right)-\left(\rho_{1}-\rho_{2}\right) \rho_{3}=\left(\rho_{1}-\rho_{2}\right)\left(1-\rho_{3}\right)>0$. In other words, the diseases are transferred from virtual $X$ to real $K_{X}$ along the killing relationship order transmission.

By Definition 3.2, the relation disease between virtual $x$ and virtual $X_{K}$ is rare since the relation cannot destroy the killing order from $X$ and $X_{K}$ although virtual $x$ cannot kill the virtual $X_{K}$. The rare relation disease between virtual $x$ and virtual $X_{K}$ makes that the killing order from $X$ to $X_{K}$ will be cured difficultly. Although rare refractory disease, but their disease transmission is natural since they are all virtual.

By Definition 3.2, the relation disease between virtual $X_{K}$ and virtual $s_{X}$, or between virtual $s_{X}$ and virtual $X_{S}$, is also rare. Thus the disease transmission from virtual $X_{K}$ to virtual $S_{X}$, or, from virtual $s_{X}$ to virtual $X_{S}$, is also natural since they are all virtual.

Only the relation disease between virtual $X_{S}$ and real $K_{X}$ is more serious since virtual $X_{S}$ cannot kill real $K_{X}$, which will destroy the killing order from $X_{S}$ to $K_{X}$ of the Yin Yang Wu Xing system if the disease continues to develop.

Finally the less serious relation disease between real $K_{X}$ and virtual $x$ makes that the killing order from $K_{x}$ to $x$ will be restored easily. The transferring way of strengthening the killing relationship by the second physiological system can make the new virtual disease of $x$ remission since virtual $X$ will get the positive increment $\Delta \varphi(X)_{4}^{1}>\left[\left(1-\rho_{2}\right)+\rho_{3}\left(\rho_{1}-\rho_{2}\right)+\left(\rho_{1}-\rho_{3}\right)\right] \Delta>0$, if $\Delta_{1} \geq \Delta>0$. 
Despite of the positive way, the system still will be for the next round of disease transmission, until the disease rehabilitation.

(2). If a subsystem $x$ of a steady multilateral system $V$ falls a real disease, denoted by $\uparrow^{0}$, which means that there is a force such that its energy $\varphi(X)$ increases, i.e., changed by the increment $\Delta \varphi(X)=\Delta>0$. By the Intervention Rule in [20], its capability of intervention reaction can make the son $X_{S}$ of $x$ to fall a real disease by a large increment $\Delta \varphi\left(X_{S}\right)=\rho_{1} \Delta>0$, denoted by $\uparrow^{+}$, and also can make the mother $s_{X}$ of $x$ to fall a real disease by a little increment $\Delta \varphi\left(S_{X}\right)=\rho_{2} \Delta>0$, denoted by $\uparrow^{-}$. Diseases of the loving subsystems have the same direction of $x$.

At the same time, its capability of intervention reaction can make the prisoner $X_{K}$ of $x$ to fall a virtual disease by a negative increment $\Delta \varphi\left(X_{K}\right)=-\rho_{1} \Delta<0$, in which its absolute is large, denoted by $\downarrow^{+}$, and also can make the bane $K_{x}$ of $x$ to fall a virtual disease by a negative increment $\Delta \varphi\left(K_{X}\right)=-\rho_{2} \Delta<0$, in which its absolute is small, denoted by $\downarrow^{-}$. Diseases of the killing subsystems are the opposite direction of $x$.

The prisoner $X_{K}$ of $x$ is the biggest victim since its increment $\Delta \varphi\left(X_{K}\right)=-\rho_{1} \Delta<0$ is the scope of big changes.

By Corollary 2.1 and Theorem 2.1, the PPI inflation rate $x \bar{\in}[a, b]$ makes that $\rho_{0}>\rho_{1}=\rho(x)>0$, such that $\left(\rho_{1}+\rho_{2} \rho_{3}\right)<\left(1-\rho_{2} \rho_{3}\right)$. In this case, the steady multilateral system $V$ is abnormal or unhealthy. The unhealthy economy implies the lack of self-protection ability. Thus the selfprotection coefficient $\rho_{3}=c \rho(x)$ will near to small such that firstly $0<\left(\rho_{1}-\rho_{3}\right)=\rho(x)(1-c) \rightarrow \rho(x)=\rho_{1}$, and secondly $0<\left(\rho_{2}-\rho_{1} \rho_{3}\right)=\rho(x)^{2}(1-c) \rightarrow \rho(x)^{2}=\rho_{2}$, i.e., $c \rightarrow 0$, if the disease continues to develop.

Consider in the worst state: $\rho_{3}=c \rho(x) \rightarrow 0$, i.e., $c \rightarrow 0$, where $\rho_{0}>\rho_{1}=\rho(x)>\rho_{2}=\rho(x)^{2}>0$. Its capability of self-protection cannot make the biggest victim as the prisoner $X_{K}$ of $x$ be restored.

In mathematics, from Theorems 3.1 and 3.2 in [20], the final increments can be obtained as follows:

$\Delta \varphi(X)_{2}=\Delta \varphi(X)_{1}+\Delta \varphi(X)=\left(1-\rho_{2} \rho_{3}\right) \Delta=\left(1-c \rho(x)^{3}\right) \Delta \rightarrow \Delta>0$,

$\Delta \varphi\left(X_{S}\right)_{2}=\Delta \varphi\left(X_{S}\right)_{1}+\Delta \varphi\left(X_{S}\right)=\left(\rho_{1}+\rho_{1} \rho_{3}\right) \Delta=\left(\rho(x)+c \rho(x)^{2}\right) \Delta \rightarrow \rho_{1} \Delta>0$,

$\Delta \varphi\left(X_{K}\right)_{2}=\Delta \varphi\left(X_{K}\right)_{1}+\Delta \varphi\left(X_{K}\right)=-\left(\rho_{1}-\rho_{3}\right) \Delta=-\rho(x)(1-c) \Delta \rightarrow-\rho(x) \Delta=-\rho_{1} \Delta<0$,

$\Delta \varphi\left(K_{X}\right)_{2}=\Delta \varphi\left(K_{X}\right)_{1}+\Delta \varphi\left(K_{X}\right)=-\left(\rho_{2}-\rho_{1} \rho_{3}\right) \Delta=-\rho(x)^{2}(1-c) \Delta \rightarrow-\rho(x)^{2} \Delta=-\rho_{2} \Delta>0$,

$\Delta \varphi\left(S_{X}\right)_{2}=\Delta \varphi\left(S_{X}\right)_{1}+\Delta \varphi\left(S_{X}\right)=\left(\rho_{2}-\rho_{1} \rho_{3}\right) \Delta=\rho(x)^{2}(1-c) \Delta \rightarrow \rho(x)^{2} \Delta=\rho_{2} \Delta>0$.

It means that the first physiological system of the steady multilateral system runs hardly, such that the final increments of self-protection are similar to the increments of intervention reaction. The hard running is not only because the killing order has been destroyed, but also the loving order cannot be running.

From the idea of TCE, by the Affording Rule, the second physiological system of the steady multilateral system will work. It is because a steady multilateral system would become ill or dies if its Chi or energy in the steady multilateral system is imbalanced or exhausted. The purpose of the second physiological system working is to increase the energy of the system, and restores the order of the killing relationship of the Yin Yang Wu Xing system.

By Theorems 3.3 and 3.4 and Corollary 3.2 in [20], the method of "Strong inhibition of the same time, support the weak", i.e., to decrease energy of real $x$ while to increase energy of virtual $K_{x}$, 
should mainly be used in this case: $x \in[a, b]$ which is equivalent to that $\rho_{0}>\rho_{1}=\rho(x)>0$, such that $\left(\rho_{1}+\rho_{2} \rho_{3}\right)<\left(1-\rho_{2} \rho_{3}\right)$. It is because the inequality makes the increment $\left(1-\rho_{2} \rho_{3}\right) \Delta_{1}$ becoming large. $\left(\rho_{1}+\rho_{2} \rho_{3}\right)<\left(1-\rho_{2} \rho_{3}\right)$ From Affording Rule, this is just the working method of the second physiological system.

By Definitions 3.1 and 3.2, consider the lack of self-protection ability, from Figure 5, for a real $x$ , its capability of intervention reaction will make the many relation diseases occurrence. Only the relation disease between real $x$ and virtual $K_{X}$ can be considered as more serious, since virtual $K_{X}$ cannot kill real $x$ which will destroy the killing order from $K_{X}$ to $x$ of the Yin Yang Wu Xing system if the disease continues to develop.

By Affording Rule again, abiding by the treatment principle of "Strong inhibition of the same time, support the weak", the intervention of the second physiological system is to decrease energy of real $x$ while increase energy of virtual $K_{x}$. If an intervention force on the subsystem $x$ of the steady multilateral system $v$ is implemented such that its energy $\varphi(X)$ has been changed by an increment $\Delta \varphi(X)=-\Delta_{1}<0$, denoted by $\Downarrow^{0}$, and at the same time, another intervention force on the subsystem $K_{x}$ of the steady multilateral system $v$ is also implemented such that its energy $\varphi\left(K_{X}\right)$ has been changed by an increment $\Delta \varphi\left(K_{X}\right)=\Delta_{1}>0$, denoted by $\Uparrow^{0}$, then from Theorem 3.4 in [20], all other subsystems: $X_{K}$ and $X_{S}$ can be restored at the same time, and the subsystems $X$ and $K_{x}$ will decrease and increase their energies by the same size but the direction opposite.

In mathematics, from Theorems 3.1, 3.3 and 3.4 in [20] , the final increments can be obtained as follows:

$\Delta \varphi(X)_{3}^{1}=-\left[\left(1-\rho_{2} \rho_{3}\right)+\left(\rho_{1}-\rho_{3}\right)\right] \Delta_{1}=-\left[\left(1-c \rho(x)^{3}\right)+\rho(x)(1-c)\right] \Delta_{1}<0$,

$\Delta \varphi\left(S_{X}\right)_{3}^{1}=\left[-\left(\rho_{1}+\rho_{2} \rho_{3}\right)+\left(\rho_{1}+\rho_{2} \rho_{3}\right)\right] \Delta_{1}=0$,

$\Delta \varphi\left(K_{X}\right)_{3}^{1}=\left[\left(1-\rho_{2} \rho_{3}\right)+\left(\rho_{1}-\rho_{3}\right)\right] \Delta_{1}=\left[\left(1-c \rho(x)^{3}\right)+\rho(x)(1-c)\right] \Delta_{1}>0$,

$\Delta \varphi\left(X_{K}\right)_{3}^{1}=\left[\left(\rho_{2}-\rho_{1} \rho_{3}\right)-\left(\rho_{2}-\rho_{1} \rho_{3}\right)\right] \Delta_{1}=0$,

$\Delta \varphi\left(X_{S}\right)_{3}^{1}=\left[-\left(\rho_{2}-\rho_{1} \rho_{3}\right)+\left(\rho_{2}-\rho_{1} \rho_{3}\right)\right] \Delta_{1}=0$.

All these changes consist of the following Figure 5.

Of course, the real disease of $x$ comes from the mother $s_{x}$ of $x$ since any real disease always comes from its mother by Theorem 3.2 in [20].

In order to cure the real $x$, by the Affording Rule, the intervention of the second physiological system must make the increment

$\Delta \varphi(X)_{3}=-\left(1-\rho_{2} \rho_{3}\right) \Delta_{1}=-\left(1-c \rho(x)^{3}\right) \Delta_{1}$

tending to be very small, for example $\Delta_{1} \geq \Delta$, which means righteousness to defeat evil, this treatment makes the increment $\Delta \varphi\left(K_{X}\right)_{3}=\left(1-\rho_{2} \rho_{3}\right) \Delta_{1}=\left(1-c \rho(x)^{3}\right) \Delta_{1}$ tending to be very big.

In fact, by Affording Rule and from Theorem 3.1 of [20], the all final increments are as follows 
$\Delta \varphi\left(K_{X}\right)_{4}^{1}=\Delta \varphi\left(K_{X}\right)_{3}^{1}+\varphi\left(K_{X}\right)_{2}=\left[\left(1-\rho_{2} \rho_{3}\right)+\left(\rho_{1}-\rho_{3}\right)\right] \Delta_{1}+\left(\rho_{2}-\rho_{1} \rho_{3}\right) \Delta$

$\geq\left[\left(1-\rho_{2} \rho_{3}\right)+\left(\rho_{1}-\rho_{3}\right)-\left(\rho_{2}-\rho_{1} \rho_{3}\right)\right] \Delta=\left[\left(1-\rho_{2}\right)+\rho_{3}\left(\rho_{1}-\rho_{2}\right)+\left(\rho_{1}-\rho_{3}\right)\right] \Delta>0$,

$\Delta \varphi\left(X_{S}\right)_{4}^{1}=\Delta \varphi\left(X_{S}\right)_{3}^{1}+\varphi\left(X_{S}\right)_{2}=\left(\rho_{1}+\rho_{2} \rho_{3}\right) \Delta=\left(\rho(x)+c \rho(x)^{2}\right) \Delta \rightarrow \rho_{1} \Delta>0$,

$\Delta \varphi\left(S_{X}\right)_{4}^{1}=\Delta \varphi\left(S_{X}\right)_{3}^{1}+\varphi\left(S_{X}\right)_{2}=\left(\rho_{2}-\rho_{1} \rho_{3}\right) \Delta=\rho(x)^{2}(1-c) \Delta \rightarrow \rho(x)^{2} \Delta=\rho_{2} \Delta>0$,

$\Delta \varphi\left(X_{K}\right)_{4}^{1}=\Delta \varphi\left(X_{K}\right)_{3}^{1}+\varphi\left(X_{K}\right)_{2}=-\left(\rho_{2}-\rho_{2} \rho_{3}\right) \Delta=-\rho(x)(1-c) \Delta \rightarrow-\rho(x) \Delta=-\rho_{1} \Delta<0$,

$\Delta \varphi(X)_{4}^{1}=\Delta \varphi(X)_{3}^{1}+\varphi(X)_{2}=-\left[\left(1-\rho_{2} \rho_{3}\right)+\left(\rho_{1}-\rho_{3}\right)\right] \Delta_{1}+\left(\rho_{2}-\rho_{1} \rho_{3}\right) \Delta$

$\leq-\left[\left(1-\rho_{2} \rho_{3}\right)\left(\rho_{1}-\rho_{3}\right)-\left(\rho_{2}-\rho_{1} \rho_{3}\right)\right] \Delta=-\left[\left(1-\rho_{2}\right)+\rho_{3}\left(\rho_{1}-\rho_{2}\right)+\left(\rho_{1}-\rho_{3}\right)\right] \Delta<0$.

Therefore, the transfer law is the first occurrence of the real disease of the bane $K_{X}$ of $x$; secondly the real disease of the son $X_{S}$ of $x$, thirdly the real disease of the mother $S_{x}$ of $x$, and finally the virtual disease of the prisoner $X_{K}$ of $x$.

In fact, watching Figure 5, $K_{X}$ is real, $X_{S}$ is real, $s_{X}$ is real, and $X_{K}$ is virtual. The order of the transfer law of diseases must be against the killing relationship order transmission. It is because

$\left[\left(1-\rho_{2}\right)+\rho_{3}\left(\rho_{1}-\rho_{2}\right)+\left(\rho_{1}-\rho_{3}\right)\right]>\left(\rho_{1}+\rho_{2} \rho_{3}\right)>\left(\rho_{2}-\rho_{1} \rho_{3}\right)>-\left(\rho_{2}-\rho_{1} \rho_{3}\right)$

$>-\left[\left(1-\rho_{2}\right)+\rho_{3}\left(\rho_{1}-\rho_{2}\right)+\left(\rho_{1}-\rho_{3}\right)\right]$.

The result is obtained from $\left(1-\rho_{2} \rho_{3}\right)>\left(\rho_{1}+\rho_{2} \rho_{3}\right)$ since

$\left[\left(1-\rho_{2}\right)+\rho_{3}\left(\rho_{1}-\rho_{2}\right)+\left(\rho_{1}-\rho_{3}\right)\right]-\left(\rho_{1}+\rho_{2} \rho_{3}\right)=1-\rho_{2} \rho_{3}+\rho_{3} \rho_{1}-\rho_{2} \rho_{3}-\rho_{2}$

$>\rho_{1}+\rho_{2} \rho_{3}+\rho_{3} \rho_{1}-\rho_{2} \rho_{3}-\rho_{2}=\left(\rho_{1}-\rho_{2}\right)+\rho_{3} \rho_{1}>0$,

and

$\left(\rho_{1}+\rho_{2} \rho_{3}\right)-\left(\rho_{2}-\rho_{1} \rho_{3}\right)=\left(\rho_{1}-\rho_{2}\right)-\left(\rho_{1}-\rho_{2}\right) \rho_{3}=\left(\rho_{1}-\rho_{2}\right)\left(1-\rho_{3}\right)>0$.

In other words, the diseases are transferred from real $x$ to virtual $X_{k}$ against the killing relationship order transmission.

By Definition 3.2, the relation disease between real $x$ and real $K_{x}$ is rare since the relation disease cannot destroy the order of the killing relationship from $K_{x}$ to $x$ although the real $K_{x}$ cannot kill the real $x$. The rare relation disease between real $x$ and real $X_{K}$ makes that the killing order from $x$ to $K_{x}$ will be cured difficultly. Although rare refractory disease, but their disease transmission is natural since they are all virtual.

By Definition 3.2, the relation disease between real $K_{X}$ and real $X_{S}$, or between real $X_{S}$ and real $S_{X}$, is also rare. Thus the disease transmission from real $K_{X}$ to real $X_{S}$, or from real $X_{S}$ to real $S_{X}$, is also natural since they are all virtual.

Only the relation disease between real $s_{X}$ and virtual $X_{K}$ is more serious since virtual $X_{K}$ cannot kill real $s_{X}$, which will destroy the killing order from $X_{K}$ to $s_{X}$ of the Yin Yang Wu Xing system if the disease continues to develop.

But the less serious relation disease between virtual $X_{K}$ and real $x$ makes that the killing order from $x$ to $X_{k}$ will be restored easily. The transferring way of strengthening the killing relationship by the second physiological system can make the new real disease of $x$ remission since real $x$ will get a negative increment

$\Delta \varphi(X)_{4}^{1}<-\left[\left(1-\rho_{2}\right)+\rho_{3}\left(\rho_{1}-\rho_{2}\right)+\left(\rho_{1}-\rho_{3}\right)\right] \Delta<0$ if $\Delta_{1} \geq \Delta>0$. 
Despite of the positive way, the system will be still for the next round of disease transmission, until the disease rehabilitation. This completes the proof.\#

Proof of Theorem 4.1. Let

$a=7.7473 \%, b=18.460 \%, t_{0}=12.269 \%$.

(1). If $x<a$, then from Theorem 2.1 and Corollary 2.1, there is that $0<\rho_{1}=\rho(x)<\rho_{0}$ which means that the economy is unhealthy where $0 \leq c \leq 1$ and $\left(\rho_{1}+\rho_{2} \rho_{3}\right)<\left(1-\rho_{2} \rho_{3}\right)$. Since $x<a$ means that the PPI inflation rate is too lower than the normal state, thus the subsystem $x$ has encountered the virtual disease.

From Theorem 3.2 and Corollary 3.2 in [20], the subsystem $x$ was considered as virtual. When the capability of self-protection is nearly to the best state, i.e., $\rho_{3}=c \rho(x)$ where $c \rightarrow 1$, if you increase the energy of the subsystem $x$ directly, then its capabilities of both intervention reaction and self-protection can make the subsystems $S_{X}, K_{X}$ and $X_{K}$ restored at the same time, but the subsystems $x$ and $X_{S}$ will increase their energies, i.e., changed by the increments $\Delta \varphi(X)_{2} \rightarrow\left(1-\rho(x)^{3}\right) \Delta>0, \Delta \varphi\left(X_{S}\right)_{2} \rightarrow\left(\rho(x)+\rho(x)^{3}\right) \Delta>0$,

respectively. Since the PPI inflation rate $x<a$ makes $0 \leq \rho_{1}=\rho(x)<\rho_{0}$ such that $\left(\rho(x)+\rho(x)^{3}\right)<\left(1-\rho(x)^{3}\right)$, therefore the subsystem $x$ can obtain the large increment $\Delta \varphi(X)_{2}=\left(1-\rho(x)^{3}\right) \Delta>0$ and the subsystem $X_{S}$ will get the small increment $\Delta \varphi\left(X_{S}\right)_{2}=\left(\rho(x)+\rho(x)^{3}\right) \Delta>0$. The later is the pseudo side effects issue, by Attaining Rule, which is just the food of the second physiological system of the steady multilateral system since that the economy is unhealthy, virtual and $x$ intervened makes that the second physiological system will attain the Chi or energy (Yang energy) from the son $x_{S}$ of the intervened subsystem $x$. The attaining way is an indirect treating for an unhealthy economic subsystem $x$ with a virtual disease.

Thus the primary treatment should be to increase the energy of the subsystem $x$ directly in order to avoid the side effects issue occurrence for an unhealthy economy. This is just one of the affording working principle of the second physiological system by Affording Rule. The idea means that "Virtual disease with an unhealthy economy is to fill itself".

When the capability of self-protection is nearly to the worst state, i.e., $\rho_{3}=c \rho(x) \rightarrow 0$ where $c \rightarrow 0$, the first physiological system will stop to work, or, the first transfer law will stop to run, while the second physiological system will begin to work, or, the second transfer law will begin to run.

By Affording Rule and the second transfer law in Theorem 3.3, the purpose of the second physiological system working is to improve the physical ability of self-protection of the first physiological system, i.e., to make all of both $\rho_{1}-\rho_{3}=\rho(x)(1-c)$ and $\rho_{2}-\rho_{1} \rho_{3}=\rho(x)^{2}(1-c)$ tending to be small, i.e., for fixed $\rho(x), c$ tends to be large, at least, greater than zero, to drive the first physiological systems will begin to work.

By the second transfer law in Theorem 3.3, for a virtual $x$, the more serious relation disease is the relation between virtual $X_{S}$ and real $K_{X}$ since virtual $X_{S}$ cannot kill real $K_{X}$ which can destroy the killing order between $X_{S}$ and $K_{X}$ if the disease continues to develop. 
Abiding by the TCE's idea of "Don't have disease cure cure non-ill", in order to avoid the more serious relation disease occurrence, it should be intervened when the intervened system was non-ill, but the incidence of second transfer law in Theorem 3.3 by this system will be to occur the more serious relation disease.

According to the intervening principle of "Strong inhibition of the same time, support the weak" from Theorem 3.4 in [20], it can be gotten that the secondary treatment is to increase the energy of $X_{S}$, and at the same time, to decrease the energy of $K_{X}$.

In general, although the economy is unhealthy and virtual, the capability of self-protection can be any state, i.e., $\rho_{3}=c \rho(x) \geq 0$ where $0 \leq c \leq 1$. The primary treatment is mainly for the normal state of the capability of self-protection, i.e., $0<\rho_{3}=c \rho(x) \rightarrow \rho(x)=\rho_{1}, \rho_{0}>\rho_{1}=\rho(x)>0$

where $0<c \leq 1$ and $\left(\rho_{1}+\rho_{2} \rho_{3}\right)<\left(1-\rho_{2} \rho_{3}\right)$. The secondary treatment is for the worst state of the capability of self-protection, i.e.,

$0 \leftarrow \rho_{3}=c \rho(x)<\rho(x)=\rho_{1}, \rho_{0}>\rho_{1}=\rho(x)>0$

where $c \rightarrow 0$ and $\left(\rho_{1}+\rho_{2} \rho_{3}\right)<\left(1-\rho_{2} \rho_{3}\right)$. Comprehensive use of the two methods for the unhealthy economy with a virtual disease can deal with a general situation, i.e., $0 \leq \rho_{3}=c \rho(x) \leq \rho(x)=\rho_{1}, \rho_{0}>\rho_{1}=\rho(x)>0$

where $0 \leq c \leq 1$ and $\left(\rho_{1}+\rho_{2} \rho_{3}\right)<\left(1-\rho_{2} \rho_{3}\right)$.\#

(2). If $a \leq x<t_{0}$, then from Theorem 2.1 and Corollary 2.1, there is $1 \geq \rho_{1}=\rho(x) \geq \rho_{0}$ which means that the economy is healthy where in general $1 \geq c \geq \frac{1-\rho(x)}{2 \rho(x)^{3}} \geq 0$ such that $\left(\rho_{1}+\rho_{2} \rho_{3}\right) \geq\left(1-\rho_{2} \rho_{3}\right)$

and $1 \geq \frac{1-\rho(x)}{2 \rho(x)^{3}}>c \geq 0$ such that $\left(\rho_{1}+\rho_{2} \rho_{3}\right) \approx\left(1-\rho_{2} \rho_{3}\right)$, i.e., $\left|\left(\rho_{1}+\rho_{2} \rho_{3}\right)-\left(1-\rho_{2} \rho_{3}\right)\right| \leq 2 \rho_{0}^{3}=0.41024$.

Since $a \leq x<t_{0}$ means that the PPI inflation rate is lower than the center $t_{0}$, thus the subsystem $x$ will encounter a virtual disease if the subsystem $x$ falls ill.

From Theorem 3.2 and Corollary 3.2 in [20], the subsystem $x$ will be considered as virtual. First the case is studied that the capability of self-protection is in the best state, i.e., $\rho_{3}=c \rho(x) \rightarrow \rho(x)=\rho_{1}>0$ where $c \rightarrow 1$. If the energy of the subsystem $s_{x}$ is intervened to increase its energy, then its capabilities of intervention reaction and self-protection can make the subsystems $K_{x}, X_{K}$ and $X_{S}$ restored at the same time, but the subsystems $s_{X}$ and $x$ will increase their energies, i.e., changed by the increments

$\Delta \varphi\left(S_{X}\right)_{2} \rightarrow\left(1-\rho(x)^{3}\right) \Delta>0, \Delta \varphi(X)_{2} \rightarrow\left(\rho(x)+\rho(x)^{3}\right) \Delta>0$,

respectively. Since the PPI inflation rate $a \leq x<t_{0}$ makes $1 \geq \rho_{1}=\rho(x) \geq \rho_{0}$ such that mainly $\left(\rho(x)+\rho(x)^{3}\right) \geq\left(1-\rho(x)^{3}\right)$, therefore the subsystem $x$ can obtain the large increment $\Delta \varphi(X)_{2}=\left(\rho(x)+\rho(x)^{3}\right) \Delta>0$ and the subsystem $S_{X}$ will get the small increment $\Delta \varphi\left(S_{X}\right)_{2}=\left(1-\rho(x)^{3}\right) \Delta$. The later is the pseudo side effects issue, by Attaining Rule, which is just the food of the second physiological system of the steady multilateral system since that the economy is healthy, virtual and $s_{x}$ intervened makes that the second physiological system will attain the Chi or 
energy (Yang energy) from the intervened subsystem $s_{x}$ directly for a healthy economy with a virtual disease.

Thus the primary treatment should be gotten to increase the energy of the subsystem $s_{x}$ in order to avoid the side effects issue occurrence for a healthy economy. The idea means that "Virtual disease with a healthy economy is to fill its mother".

Since the economy is healthy, the worst state of the capability of self-protection will not be possible, i.e., $\rho_{3}=c \rho(x)>0$ where $0<c \leq 1$, the first physiological system will not stop to work, or, the first transfer law will not stop to run, while the second transfer law will not begin to run. Is the first physiological system working in order to improve its corresponding intervention ability, at the same time, to provide the food for the second physiological system. In other words, it makes $\rho_{1}+\rho_{2} \rho_{3}=\rho(x)+c \rho(x)^{3}$ tending to be large while $1-\rho_{2} \rho_{3}=\left(1-c \rho(x)^{3}\right)$ tending to be small, i.e., $\rho(x)$ tends to large while $c$ also tends to be large, the best, equal to 1 , such that the non-existence of side effects, or, the non-existence of medical and drug resistance problem. The work mainly improves the loving relationship since the loving relationship is mainly used. By the first transfer law in Theorem 3.2 for a virtual $x$, the more serious relation disease is the relation between virtual $x$ and real $X_{K}$ since virtual $x$ cannot kill the real $X_{K}$ which can destroy the killing order between $x$ and $X_{K}$ if the disease continues to develop.

Abiding by the TCE's idea of "Don't have disease cure cure non-ill", in order to avoid the more serious relation disease occurrence, it should be intervened when the intervened system was non-disease, but the incidence of the first transfer law in Theorem 3.2 by this system will be to occur the more serious relation disease.

According to the intervening principle of "Strong inhibition of the same time, support the weak" from Theorem 3.4 in [20], the secondary treatment is gotten to increase the energy of $x$, and at the same time, to decrease the energy of $X_{K}$.

In general, since the economy is healthy and virtual, the capability of self-protection cannot be the worst state and should be normal, i.e., $\rho_{3}=c \rho(x)>0$ where $0<c \leq 1$. The primary treatment is for the best state of the capability of self-protection, i.e.,

$0<\rho_{3}=c \rho(x) \rightarrow \rho(x)=\rho_{1}, \rho_{0} \leq \rho_{1}=\rho(x) \leq 1$

where $c \rightarrow 1$ and $\left(\rho_{1}+\rho_{2} \rho_{3}\right) \geq\left(1-\rho_{2} \rho_{3}\right)$. The secondary treatment is for the normal state of the capability of self-protection, i.e., $0<\rho_{3}=c \rho(x)<\rho(x)=\rho_{1}, \rho_{0} \leq \rho_{1}=\rho(x) \leq 1$

where $0<c<1$ and $\left(\rho_{1}+\rho_{2} \rho_{3}\right) \geq\left(1-\rho_{2} \rho_{3}\right)$. Comprehensive use of the two methods for the healthy economy with a virtual disease can deal with a general situation, i.e., $0<\rho_{3}=c \rho(x) \leq \rho(x)=\rho_{1}, \rho_{0} \leq \rho_{1}=\rho(x) \leq 1$

where $0<c \leq 1$ and $\left(\rho_{1}+\rho_{2} \rho_{3}\right) \geq\left(1-\rho_{2} \rho_{3}\right)$.\# 
(3). If $t_{0} \leq x \leq b$, then from Theorem 2.1 and Corollary 2.1, there is $1 \geq \rho_{1}=\rho(x) \geq \rho_{0}$ which means that the economy is healthy where in general $1 \geq c \geq \frac{1-\rho(x)}{2 \rho(x)^{3}} \geq 0$ such that $\left(\rho_{1}+\rho_{2} \rho_{3}\right) \geq\left(1-\rho_{2} \rho_{3}\right)$

and $1 \geq \frac{1-\rho(x)}{2 \rho(x)^{3}}>c \geq 0$ such that $\left(\rho_{1}+\rho_{2} \rho_{3}\right) \approx\left(1-\rho_{2} \rho_{3}\right)$, i.e., $\left|\left(\rho_{1}+\rho_{2} \rho_{3}\right)-\left(1-\rho_{2} \rho_{3}\right)\right|<2 \rho_{0}^{3}=0.41024$.

Since $t_{0} \leq x \leq b$ means that the PPI inflation rate is higher than or equal to the center $t_{0}$, thus the subsystem $x$ will encounter a real disease if the subsystem $x$ falls ill.

From Theorem 3.2 and Corollary 3.2 in [20] , the subsystem $X$ will be considered as real. First the case is studied that the capability of self-protection is in the best state, i.e., $\rho_{3}=c \rho(x) \rightarrow \rho(x)=\rho_{1}$ where $c \rightarrow 1$. If the energy of the subsystem $X_{S}$ is intervened to decrease its energy, then its capabilities of intervention reaction and self-protection can make the subsystems $X_{K}, K_{X}$ and $S_{X}$ restored at the same time, but the subsystems $x$ and $X_{S}$ will decrease their energies, i.e., changed by the increments

$\Delta \varphi(X)_{2} \rightarrow-\left(\rho(x)+\rho(x)^{3}\right) \Delta>0, \Delta \varphi\left(X_{S}\right)_{2} \rightarrow-\left(1-\rho(x)^{3}\right) \Delta>0$,

respectively. Since the PPI inflation rate $t_{0} \leq x \leq b$ makes $1 \geq \rho_{1}=\rho(x) \geq \rho_{0}$ such that mainly $\left(\rho(x)+\rho(x)^{3}\right) \geq\left(1-\rho(x)^{3}\right)$, therefore the subsystem $X$ can obtain the substantial reduction $\Delta \varphi(X)_{2}=-\left(\rho(x)+\rho(x)^{3}\right) \Delta<0$ and the subsystem $X_{S}$ will get a modest reduction $\Delta \varphi\left(X_{S}\right)_{2}=-\left(1-\rho(x)^{3}\right) \Delta<0$. The later is the pseudo side effects issue, by Attaining Rule, which is just the food of the second physiological system of the steady multilateral system since that the economy is healthy, real and $X_{S}$ intervened makes that the second physiological system will attain the Chi or energy (Yin energy) from the intervened subsystem $X_{S}$ directly for a healthy economy with a real disease.

Thus the primary treatment should be gotten to decrease the energy of the subsystem $X_{S}$ in order to avoid the side effects issue occurrence for a healthy economy. The idea means that "Real disease with a healthy economy is to rush down its son".

Since the economy is healthy, the state of the capability of self-protection should be normal, i.e., $\rho_{3}=c \rho(x)>0$ where $0<c \leq 1$. In this case, the first physiological system will not stop to work, or, the first transfer law will not stop to run, while the second transfer law will not begin to run.

Is the first physiological system working in order to improve its corresponding intervention ability, at the same time, to provide the food for the second physiological system. In other words, it makes $\rho_{1}+\rho_{2} \rho_{3}=\rho(x)+c \rho(x)^{3}$ tending to be large while $1-\rho_{2} \rho_{3}=\left(1-c \rho(x)^{3}\right)$ tending to be small. Equivalently, the way can make $\rho(x)$ tending to large and $c$ also tending to be large, the best, equal to 1 , such that the non-existence of side effects issue, or, the non-existence of medical and drug resistance problem. The work mainly improves the loving relationship since the loving relationship is mainly used.

By the first transfer law in Theorem 3.2, the more serious relation disease is the relation between virtual $K_{x}$ and real $x$ since virtual $K_{x}$ cannot kill the real $x$ which can destroy the killing order between $K_{X}$ and $x$ if the disease continues to develop. 
Abiding by the TCE's idea of "Don't have disease cure cure non-ill", in order to avoid the more serious relation disease occurrence, it should be intervened when the intervened system was non-disease, but the incidence of first transfer law in Theorem 3.2 by this system will be to occur the more serious relation disease.

According to the intervening principle of "Strong inhibition of the same time, support the weak" from Theorem 3.4 in [20], the secondary treatment is gotten to increase the energy of $K_{X}$, and at the same time, to decrease the energy of $x$.

In general, since the economy is healthy with an expected real disease, the capability of selfprotection should be normal, i.e., $\rho_{3}=c \rho(x)>0$ where $0<c \leq 1$. The primary treatment is for the best state of the capability of self-protection, i.e.,

$0<\rho_{3}=c \rho(x) \rightarrow \rho(x)=\rho_{1}, \rho_{0} \leq \rho_{1}=\rho(x) \leq 1$

where $c \rightarrow 1$ and $\left(\rho_{1}+\rho_{2} \rho_{3}\right) \geq\left(1-\rho_{2} \rho_{3}\right)$. The secondary treatment is for the normal state of the capability of self-protection, i.e., $\quad 0<\rho_{3}=c \rho(x)<\rho(x)=\rho_{1}, \rho_{0} \leq \rho_{1}=\rho(x) \leq 1$ where $0<c<1$ and $\left(\rho_{1}+\rho_{2} \rho_{3}\right) \geq\left(1-\rho_{2} \rho_{3}\right)$. Comprehensive use of the two methods for the healthy economy with a real disease can deal with a general situation, i.e., $0<\rho_{3}=c \rho(x) \leq \rho(x)=\rho_{1}, \rho_{0} \leq \rho_{1}=\rho(x) \leq 1$

where $0<c \leq 1$ and $\left(\rho_{1}+\rho_{2} \rho_{3}\right) \geq\left(1-\rho_{2} \rho_{3}\right)$.\#

(4). If $x>b$, then from Corollary 2.1, there is $0<\rho_{1}=\rho(x)<\rho_{0}$ which means that the economy is unhealthy where $0 \leq c \leq 1$ and $\left(\rho_{1}+\rho_{2} \rho_{3}\right)<\left(1-\rho_{2} \rho_{3}\right)$. Since $x>b$ means that the PPI inflation rate is too higher than the normal state, thus the subsystem $x$ has encountered the real disease. From Theorem 3.2 and Corollary 3.2 in [20], the subsystem $x$ was considered as real. When the capability of self-protection is in the best state, i.e., $\rho_{3}=c \rho(x) \rightarrow \rho(x)=\rho_{1}$ where $c \rightarrow 1$, if the energy of the subsystem $x$ is intervened to decrease its energy directly, then its capabilities of intervention reaction and self-protection can make the subsystems $X_{K}, K_{X}$ and $X_{S}$ restored at the same time, but the subsystems $x$ and $s_{x}$ will decrease their energies, i.e., changed by the increments

$\Delta \varphi(X)_{2} \rightarrow-\left(1-\rho(x)^{3}\right) \Delta<0, \Delta \varphi\left(S_{X}\right)_{2} \rightarrow-\left(\rho(x)+\rho(x)^{3}\right) \Delta<0$,

respectively. Since the PPI inflation rate $x>b$ makes $0<\rho_{1}=\rho(x)<\rho_{0}$ such that $\left(\rho(x)+\rho(x)^{3}\right)<\left(1-\rho(x)^{3}\right)$, therefore the subsystem $X$ can obtain the substantial reduction $\Delta \varphi(X)_{2}=-\left(1-\rho(x)^{3}\right) \Delta<0$ and the subsystem $S_{X}$ will get a modest reduction $\Delta \varphi\left(S_{X}\right)=-\left(\rho(x)+\rho(x)^{3}\right) \Delta<0$. The later is the pseudo side effects, by Attaining Rule, which is just the food of the second physiological system of the steady multilateral system since that the economy is unhealthy, real and $x$ intervened makes that the second physiological system will attain the Chi or energy (Yin energy) from the mother $s_{x}$ of the intervened subsystem $x$. The attaining way is an indirect treating for an unhealthy economic subsystem $x$ with a real disease. Thus the primary treatment should be gotten to decrease the energy of the subsystem $x$ directly, in order to avoid the side effects issue occurrence for an unhealthy economy. This is just one of the affording working principle of the second physiological system by Affording Rule. The idea means that "Real disease with an unhealthy economy is to rush down itself". 
When the capability of self-protection is at the worst state, i.e., $\rho_{3}=c \rho(x) \rightarrow 0$ where $c \rightarrow 0$, the first physiological system will stop to work, or, the first transfer law will stop to run, while the second physiological system will begin to work, or, the second transfer law will begin to run.

By Affording Rule and the second transfer law in Theorem 3.3, the purpose of the second physiological system working is to improve the physical ability of self-protection of the first physiological system, i.e., to make all of both $\rho_{1}-\rho_{3}=\rho(x)(1-c)$ and $\rho_{2}-\rho_{1} \rho_{3}=\rho(x)^{2}(1-c)$ tending to be small, i.e., for fixed $\rho(x), c$ tends to be large, at least, greater than zero, to drive the first physiological systems will begin to work. The work mainly improves the killing relationship since the killing relationship is mainly used.

By the second transfer law in Theorem 3.3, for a real $x$, the more serious relation disease is the relation between virtual $X_{K}$ and real $S_{X}$ since virtual $X_{K}$ cannot kill the real $S_{X}$ which can destroy the killing order between $X_{K}$ and $s_{X}$ if the disease continues to develop.

Abiding the TCE's idea of "Don't have disease cure cure non-ill", in order to avoid the more serious relation disease occurrence, it should be intervened when the intervened system was non-disease, but the incidence of second transfer law in Theorem 3.3 by this system will be to occur the more serious relation disease.

According to the intervening principle of "Strong inhibition of the same time, support the weak" from Theorem 3.4 in [20], the secondary treatment is gotten to decrease the energy of $s_{x}$, and at the same time, to increase the energy of $X_{K}$.

In general, although the economy is unhealthy with a real disease, the capability of selfprotection can be any state, i.e., $\rho_{3}=c \rho(x) \geq 0$ where $0 \leq c \leq 1$. The primary treatment is mainly for the normal state of the capability of self-protection, i.e., $0<\rho_{3}=c \rho(x) \rightarrow \rho(x)=\rho_{1}, \rho_{0}>\rho_{1}=\rho(x)>\rho_{2}=\rho(x)^{2}>0$

where $0<c \leq 1$ and $\left(\rho_{1}+\rho_{2} \rho_{3}\right)<\left(1-\rho_{2} \rho_{3}\right)$. The secondary treatment is for the worst state of the capability of self-protection, i.e., $0 \leftarrow \rho_{3}=c \rho(x)<\rho(x)=\rho_{1}, \rho_{0}>\rho_{1}=\rho(x)>\rho_{2}=\rho(x)^{2}>0$

where $c \rightarrow 0$ and $\left(\rho_{1}+\rho_{2} \rho_{3}\right)<\left(1-\rho_{2} \rho_{3}\right)$. Comprehensive use of the two methods for the unhealthy economy with a virtual disease can deal with a general situation, i.e., $0 \leq \rho_{3}=c \rho(x) \leq \rho(x)=\rho_{1}, \rho_{0}>\rho_{1}=\rho(x)>\rho_{2}=\rho(x)^{2}>0$

where $\mathbf{0} \leq \boldsymbol{c} \leq \mathbf{1}$ and $\left(\rho_{1}+\rho_{2} \rho_{3}\right)<\left(1-\rho_{2} \rho_{3}\right)$. This completes the proof. \#

\section{References}

C.W.Su, K.Khan; O.R.Lobont and H.C.Sung, "Is there any Relationship between Producer Price Index and Consumer Price Index in Slovakia? A Bootstrap Rolling Approach 1". Ekonomicky Casopis, Bratislava,08/2016,Vol.64, No.7, pp: 611-628

T.M.Crone, N.I.Leonard and V.Richard, "Rents have been rising, not falling, in the postwar period”. Journal of Economics and Behavioral Studies, 2010, Vol.92, No.3 pp: 628-642. doi:10.1162/REST_a_00015

I.Pauhofova and A.Qineti, "The basic determinants of price development in agriculture and food industry of Slovakia". Ekonomicky Casopis, 2002, Vol.50, No. 2, pp: 165-181.

M.Funke, A.Mehrotra and H.Yu, "Tracking Chinese CPI inflation in real time". Empir Econ, 2015, Vol.48, pp:16191641. doi:10.1007/s00181-014-0837-3 
A.Formica and G.Kingston, "Inflation Insurance for Australian Annuitants”. Australian Journal of Management, 12/1991, 1991, Vol.16, No.2, pp:145-163. doi:10.1177/031289629101600203

G.Fan,L.P.He and J.N.Hu, “CPI vs. PPI: Which drives which?”. Frontiers of Economics in China, 2009, Vol.4, Issue 3, pp: 317-334.

R.Adams, "US prices for most pigments have fallen since end-2012 but CPI inflation is gathering pace". Focus on Pigments, 2014, Vol.2014, Issue 3, pp:1-93. doi:10.1016/S0969-6210(14)70096-7

J.Hausman, "Cellular Telephone, New Products, and CPI". Journal of Business \& Economic Statistics, 1999, Vol.17, Issue 2, pp:188-194. doi:10.1080/07350015.1999.10524809

D.Nahm, "The Effects of New Goods and Substitution on the Korean CPI as a Measure of Cost of Living". International Economic Journal, 2015, Vol.29, No.1, pp:57-72. doi:10.1080/10168737.2014.928894

I.A.Moosa, "Does the Chinese official CPI underestimate inflation?". Applied Economics Letters, 05/1997, Vol.4, Issue 5, pp:301-304

X.Zhao,“Forecasting inflation in China”. Dissertation/Thesis, 01/2013, Carleton University (Canada)

H.D.M.Daniel,"Essays in macroeconomics and international finance”. Dissertation/Thesis, 2012, University of Maryland, College Park.b Economics.

Anonymous, "Czech National Bank's Inflation report for fourth quarter 1998”. Finance A Uver, 1999, Vol.49, No. 4, pp:189-201.

Anonymous, “The Czech National Bank's Inflation Report for the first quarter 1999”. Finance A Uver, 1999, Vol.49, No.7, pp:389-406.

Y.S.Zhang,"Multilateral Matrix Theory”. Beijing:Chinese Statistics Press, 1993.

Y.S.Zhang,"Multilateral System Theory”. http://www.mlmatrix.com, 2007.

Y.S.Zhang,"Mathematical reasoning of treatment principle based on Yin Yang Wu Xing theory in traditional Chinese medicine", Chinese Medicine, 2011, Vol.2, No.1, pp:6-15. doi:10.4236/cm.2011.21002

Y.S.Zhang, "Mathematical reasoning of treatment principle based on Yin Yang Wu Xing theory in traditional Chinese medicine (II)", Chinese Medicine, 2011, Vol.2, No.4, pp:158-170. doi:10.4236/cm.2011.24026

Y.S.Zhang, "Mathematical reasoning of treatment principle based on the stable logic analysis model of complex systems", Intelligent control and automation, 2012, Vol.3, No.1, pp:6-15. doi:10.4236/ica.2012.31001

Y.S.Zhang and W.L.Shao, "Image mathematics-mathematical intervening principle based on Yin Yang Wu Xing theory in traditional Chinese mathematics (I)", Applied Mathematics, 2012, Vol.3, No.2, pp:617-636. doi:10.4236/am.2012.36096

Z.Q.Zhang and Y.S.Zhang, "Mathematical reasoning of economic intervening principle based on Yin Yang Wu Xing theory in traditional Chinese economics (I)", Modern Economics, 2013, Vol.4, pp:130-144. doi:10.4236/me.2013.42016

N.Q.Feng,Y.H.Qiu, F.Wang, Y.S..Zhang and S.Q.Yin, "A logic analysis model about complex system's stability: enlightenment from nature". Lecture Notes in Computer Science, 2005, Vol.3644, pp:828-838. doi.org/10.1007/11538059_86

Y.S.Zhang, S.S.Mao, C.Z.Zhan and Z.G. Zheng, "Stable structure of the logic model with tow causal effects". Chinese Journal of Applied Probability and Statistics, 2005, Vol.21, No.4, pp:366-374 
Table 1. Inflation Rates of Finance, GDP, CPI,PPI,RPI,GBR and AAF

\begin{tabular}{|c|c|c|c|c|c|c|c|c|}
\hline No. & $M_{2}$ & rate & $\begin{array}{c}\text { GDP } \\
(1978=100)\end{array}$ & tate & \multicolumn{2}{|c|}{ Finance inflation rate } & $\begin{array}{c}\text { CPI } \\
(1984=100)\end{array}$ & rate \\
\hline 1990 & 15293.4 & & 18774.3 & & & 216.4 & \\
\hline 1991 & 19349.9 & 0.26525 & 21895.5 & 0.14255 & \multicolumn{2}{|c|}{0.10739} & 223.8 & 0.03307 \\
\hline 1992 & 25402.2 & 0.31278 & 27068.3 & 0.19110 & \multicolumn{2}{|c|}{0.10216} & 238.1 & 0.06006 \\
\hline 1993 & 34579.8 & 0.36129 & 35524.3 & 0.23803 & \multicolumn{2}{|c|}{0.09956} & 273.1 & 0.12816 \\
\hline 1994 & 46923.5 & 0.35696 & 48459.6 & 0.26693 & \multicolumn{2}{|c|}{0.07106} & 339.0 & 0.19440 \\
\hline 1995 & 60750.5 & 0.29467 & 61129.8 & 0.20727 & \multicolumn{2}{|c|}{0.07240} & 396.9 & 0.14588 \\
\hline 1996 & 76094.9 & 0.25258 & 71572.3 & 0.14590 & \multicolumn{2}{|c|}{0.09310} & 429.9 & 0.07676 \\
\hline 1997 & 90995.3 & $0.1958 \mathrm{I}$ & 79429.5 & 0.09892 & \multicolumn{2}{|c|}{0.08817} & 441,9 & 0.02716 \\
\hline 1998 & 104498.5 & 0.14839 & 84883.7 & 0.06425 & \multicolumn{2}{|c|}{0.07906} & 438.4 & -0.00798 \\
\hline 1999 & 119897.9 & 0.14736 & 90187,7 & $0.0588 \mathrm{I}$ & \multicolumn{2}{|c|}{0.08364} & 432.2 & -0.01435 \\
\hline 2000 & 134610.3 & 0.12271 & 99776.3 & 0.09610 & \multicolumn{2}{|c|}{0.02427} & 434.0 & 0.00415 \\
\hline 2001 & 158301.9 & 0.17600 & 110270.4 & 0.09517 & \multicolumn{2}{|c|}{0.07381} & 437.0 & 0.00686 \\
\hline 2002 & 185007.0 & 0.16870 & 121002.0 & 0.08869 & \multicolumn{2}{|c|}{0.07349} & 433.5 & -0.00807 \\
\hline 2003 & 221222.8 & 0.19575 & 136564.6 & 0.11396 & \multicolumn{2}{|c|}{0.07343} & 438.7 & 0.01185 \\
\hline 2004 & 254107.0 & 0.14865 & 160714,4 & 0.15027 & \multicolumn{2}{|c|}{-0.00141} & 455,8 & 0.03752 \\
\hline 2005 & 298755.7 & 0.17571 & 185895.8 & 0.13546 & \multicolumn{2}{|c|}{0.03545} & 464.0 & 0.01767 \\
\hline 2006 & 345577.9 & 0.15672 & 217656.6 & 0.14592 & \multicolumn{2}{|c|}{0.00943} & 471.0 & 0,01486 \\
\hline 2007 & 403442.2 & 0.16744 & $268019 \mathrm{~A}$ & 0.18791 & -0.01 & & 493.6 & 0.04579 \\
\hline 2008 & 475166.6 & 0.17778 & 316751,7 & 0.15385 & $0.02 x$ & & 522.7 & 0.05567 \\
\hline 2009 & 610224.5 & 0.28423 & 345629.2 & 0.08355 & 0.18 & & 519.0 & -0.00713 \\
\hline 2010 & 725851.8 & 0.18948 & 4089030 & 0.15474 & $0,03 x$ & & 536.1 & 0.03190 \\
\hline 2011 & 851590.9 & 0.17323 & 484123.5 & 0.15537 & 0.01 & & 565.0 & 0.05115 \\
\hline 2012 & 974148.8 & 0.14392 & 534123,0 & $0.0936 \mathrm{I}$ & 0.046 & & 579.7 & 0.02536 \\
\hline 2013 & 1106525.0 & 0.13589 & 588018.8 & 0.09166 & 0.04 & & 594.8 & 0.02539 \\
\hline 2014 & 1228374.8 & 0.11012 & 635910.0 & 0.07531 & 0.03 & & 606.7 & 0.01961 \\
\hline No. & $\begin{array}{c}\text { PPI } \\
(3984=100)\end{array}$ & rate & $\begin{array}{c}\text { RPI } \\
(1984=100]\end{array}$ & rate & GBR & rate & AAF & rate \\
\hline 1990 & 207.7 & & 159.0 & & 2937.10 & & 7662.1 & \\
\hline 1991 & 213.7 & 0,02808 & 168.9 & 0.05861 & 3149.48 & 0.07231 & 8157.0 & 0.06459 \\
\hline 1992 & 225.2 & 0.05107 & 180.4 & 0.06375 & 3483,37 & 0.10601 & 9084,7 & 0.11373 \\
\hline 1993 & 254.9 & 0.11652 & 223.7 & 0.19356 & 4348.95 & 0.24849 & 10995.5 & 0.21033 \\
\hline 1994 & 310.2 & 0.17827 & 267.3 & 0.16311 & 5218.10 & 0.19985 & 15750.5 & 0.43245 \\
\hline 1995 & 356.1 & 0.12890 & 307.1 & 0.12960 & 6242.20 & 0.19626 & 20340.9 & 0.29144 \\
\hline 1996 & 377.8 & 0.05744 & 316.0 & 0.02816 & 7407.99 & 0.18676 & 22353.7 & 0.09895 \\
\hline 1997 & 380.8 & 0.00788 & 315,0 & -.00317 & 8651.14 & 0.16781 & 23788.4 & 0.06418 \\
\hline 1998 & 370.9 & -.02669 & 302.1 & -.04270 & 9875.95 & 0.14158 & 24541.9 & 0.03168 \\
\hline 1999 & 359.8 &,- 03085 & 294.8 & -.02476 & 11444.08 & 0.15878 & 24519.1 & 0.00093 \\
\hline 2000 & 354,4 & -.01524 & 303.1 & 0.02738 & 13395.23 & 0.17049 & 24915.8 & 0.01618 \\
\hline 2001 & 351.6 & -.00796 & 299.2 & -.01303 & 16386.04 & 0.22327 & 26179.6 & 0.05072 \\
\hline 2002 & 347.0 & -01326 & 292.6 & -.02256 & 18903.64 & 0.15364 & 27390.8 & 0.04627 \\
\hline 2003 & 346.7 &,- 00087 & 299.3 & 0.02239 & 21715.25 & 0.14873 & 29691.8 & 0.08401 \\
\hline 2004 & 356.4 & 0,02722 & 317.6 & 0.05762 & 26396.47 & 0.21557 & 36239.0 & 0.22051 \\
\hline 2005 & 359.3 & 0.00807 & 333.2 & 0.04682 & 31649.29 & 0.19900 & 39450.9 & 0.08863 \\
\hline 2006 & 362.9 & 0.00992 & 343.2 & 0.02914 & 38760.20 & 0.22468 & 40810.8 & 0.03447 \\
\hline 2007 & 376.7 & 0.03663 & 353.8 & 0.02996 & 51321,78 & 0.32408 & 48893.0 & 0.19804 \\
\hline 2008 & 398.9 & 0.05565 & 378.2 & 0.06452 & 61330.35 & 0.19502 & 58002.2 & 0.18631 \\
\hline 2009 & 394,1 & -.01218 & 357.8 & -.05702 & 68518.30 & 0.11720 & 60361.0 & 0.04067 \\
\hline 2010 & 406.3 & 0.03003 & 377.5 & 0.05219 & 83101.51 & 0.21284 & 69319.8 & 0.14842 \\
\hline 2011 & 426.2 & 0.04669 & 400,2 & 0.05672 & 103874.43 & 0.24997 & 81303.9 & 0.17288 \\
\hline 2012 & 434.7 & 0.01955 & 393.4 & -.01729 & 117253.52 & 0.12880 & 89453.0 & 0.10023 \\
\hline 2013 & 440.8 & 0.01384 & 385,9 & -.01944 & 129209.64 & 0.10197 & 96995.3 & 0.08432 \\
\hline 2014 & 445.2 & 0.00988 & 378.6 & -.01928 & 140370.03 & 0.08637 & 102226.1 & 0.05393 \\
\hline $\begin{array}{l}\text { Assum } \\
\text { price in } \\
\text { Reven } \\
\text { today a } \\
\text { the inf } \\
\text { PPI is }\end{array}$ & $\begin{array}{l}\mathrm{M}_{2} \text { or } M \\
\text { (CPI) as } C \\
\text { (BR) as } G_{b} \\
\text { ast year resper } \\
\text { rate of } M_{2} \\
\left.P^{\prime}\right) / P^{\prime} \text {, the in }\end{array}$ & $\begin{array}{l}\text { as issued in } \\
\text { of } C^{\prime} \text {, the } \mathrm{Pr} \\
\text { or } G_{b} \text {, and } \mathrm{t} \\
\text { tively, the acth } \\
\text { s }\left(M_{2}-M_{2}^{\prime}\right) \\
\text { lation rate of }\end{array}$ & $\begin{array}{l}\text { the circulation o } \\
\text { oducer Price Inde } \\
\text { the total output va } \\
\text { ual need of mone } \\
M_{2} \text {, the inflation } \\
\text { RPI is }\left(R-R^{\prime}\right) / F\end{array}$ & $\begin{array}{l}\text { neralized } \\
\text { PI) as } \\
\text { of Agricu } \\
\text { real terms } \\
\text { e of GDP } \\
\text { the inflati }\end{array}$ & $\begin{array}{l}f, \text { the Gross D } \\
p^{\prime} \text {, the Retai } \\
\text { forestry Anm } \\
\text { e circulation } \\
\left.;-G^{\prime}\right) / G^{\prime} \text {, the } \\
\text { e of GBR is }\end{array}$ & $\begin{array}{l}\text { c Product } \\
\text { Index (RP } \\
\text { andry and } \\
A_{2}^{\prime} \times(G) \\
\text { in rate of } \\
M G_{b} \text {, the }\end{array}$ & $\begin{array}{l}\text { OP) as } G \text { or } \\
\text { is } R \text { or } R^{\prime} \text {, } \\
\text { hery (AAF) a } \\
\text { ) for last yez } \\
\text { is (C-C'lic' } \\
\text { flation rate of }\end{array}$ & $\begin{array}{l}\text { the consun } \\
\text { eneral Bud } \\
\text { or } A^{\prime} \text {, } \\
\text { ce level. Th } \\
\text { flation rate } \\
\text { is }\left(A-A^{\prime}\right) /\end{array}$ \\
\hline
\end{tabular}

